hep-th/0510251

\title{
An Index for 4 dimensional Super Conformal Theories
}

\author{
Justin Kinney $^{a}$, Juan Maldacena ${ }^{b}$, Shiraz Minwalla ${ }^{c, d}$ and Suvrat Raju ${ }^{d}$ \\ a Department of Physics, Princeton University, Princeton, NJ 08544, USA \\ ${ }^{b}$ Institute for Advanced Study, Princeton, NJ 08540, USA \\ c Tata Institute of Fundamental Research, Mumbai 400005, India \\ d Jefferson Physical Laboratory, Harvard University, Cambridge, MA 02138, USA
}

\begin{abstract}
We present a trace formula for an index over the spectrum of four dimensional superconformal field theories on $S^{3} \times$ time. Our index receives contributions from states invariant under at least one supercharge and captures all information - that may be obtained purely from group theory - about protected short representations in 4 dimensional superconformal field theories. In the case of the $\mathcal{N}=4$ theory our index is a function of four continuous variables. We compute it at weak coupling using gauge theory and at strong coupling by summing over the spectrum of free massless particles in $A d S_{5} \times S^{5}$ and find perfect agreement at large $N$ and small charges. Our index does not reproduce the entropy of supersymmetric black holes in $A d S_{5}$, but this is not a contradiction, as it differs qualitatively from the partition function over supersymmetric states of the $\mathcal{N}=4$ theory. We note that entropy for some small supersymmetric $A d S_{5}$ black holes may be reproduced via a D-brane counting involving giant gravitons. For big black holes we find a qualitative (but not exact) agreement with the naive counting of BPS states in the free Yang Mills theory. In this paper we also evaluate and study the partition function over the chiral ring in the $\mathcal{N}=4$ Yang Mills theory.
\end{abstract}




\section{Contents}

1. Introduction . . . . . . . . . . . . . . . . . . . . . . . . . . . . . . . . . 2

2. 4 dimensional Superconformal Algebras and their Unitary Representations . . . . . . 4

2.1. The 4 dimensional Conformal Algebra . . . . . . . . . . . . . . . . . . . . 5

2.2. Unitary Representations of the Conformal Group . . . . . . . . . . . . . . . . 6

2.3. Unitary Representations of $d=4$ Superconformal Algebras . . . . . . . . . . . 8

2.4. The Null Vectors in Short Representations . . . . . . . . . . . . . . . . . . . 11

2.5. Indices For Four Dimensional Super Conformal Algebras . . . . . . . . . . . . 12

3. A Trace Formula for the Indices of Superconformal Algebra . . . . . . . . . . . . . 13

3.1. The Commuting Subalgebra . . . . . . . . . . . . . . . . . . . . . . . . . 14

3.2. $\mathcal{I}^{W L}$ expanded in sub-algebra characters with $I^{L}$ as coefficients . . . . . . . . . 15

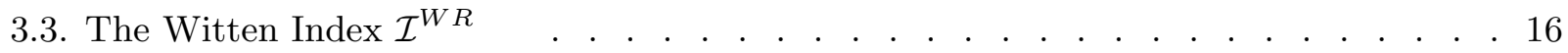

4. Computation of the Index in $\mathcal{N}=4$ Yang Mills on $S^{3}$. . . . . . . . . . . . . . . . 18

4.1. Weak Coupling . . . . . . . . . . . . . . . . . . . . . . . . . . . . . 18

4.2. Strong Coupling . . . . . . . . . . . . . . . . . . . . . . . . . . . . . . 21

5. The partition function over BPS states . . . . . . . . . . . . . . . . . . . 23

5.1. Partition Function at $\Delta=0$ in Free Yang Mills . . . . . . . . . . . . . . . . 24

5.2. Cohomology at Strong Coupling: Low energies . . . . . . . . . . . . . . . . 26

5.3. Cohomology at Strong Coupling: High Energies . . . . . . . . . . . . . . . . 28

5.4. Cohomology at Intermediate Energies: Giant Gravitons and Small Black Holes . . . 31

6. Partition Functions over $\frac{1}{2}, \frac{1}{4}^{t h}$ and $\frac{1}{8}^{t h}$ BPS States . . . . . . . . . . . . . . . . 33

6.1. Enumeration of $\frac{1}{8}^{\text {th }}$, quarter and half BPS Cohomology . . . . . . . . . . . . . 34

6.2. Protected double trace operator in the $\mathbf{2 0}$. . . . . . . . . . . . . . . . . . . . . 37

6.3. Large $N$ limits and Phase Transitions . . . . . . . . . . . . . . . . . . . . . 39

7. Discussion . . . . . . . . . . . . . . . . . . . . . . . . . . . . . . . . . . 41

Appendix A. The $d=4$ Superconformal Algebra . . . . . . . . . . . . . . . . . . . 43

A.1. The Commutation Relations . . . . . . . . . . . . . . . . . . . . . . . . 43

A.2. An Oscillator Construction of the Algebra . . . . . . . . . . . . . . . . . . 44

Appendix B. Algebraic Details Concerned with the Index . . . . . . . . . . . . . . . 45

B.1. Superconformal Indices from Joining Rules . . . . . . . . . . . . . . . . . . 45

B.2. The Index $\mathcal{I}^{W L}$ as a sum over characters . . . . . . . . . . . . . . . . . . . . 46

B.3. Representation Theory of the Subalgebra $S U(2,1 \mid m-1) \quad$. . . . . . . . . . . . 48

Appendix C. Conventions and Computations for the $\mathcal{N}=4$ Index . . . . . . . . . . . 49

C.1. Weights of the Supercharges . . . . . . . . . . . . . . . . . . . . . . . . . 49

C.2. Racah Speiser Algorithm . . . . . . . . . . . . . . . . . . . . . . . . . . 50

C.3. State content of 'graviton' representations . . . . . . . . . . . . . . . . . . . 50

C.4. Character of $S U(3) \quad$. . . . . . . . . . . . . . . . . . . . . . . . . . . . 52

C.5. Translation between bases . . . . . . . . . . . . . . . . . . . . . . . . . 52

C.6. Index on the Fock space . . . . . . . . . . . . . . . . . . . . . . . . . . 53

Appendix D. Comparison of the Cohomological Partition Function and the Index . . . . . 53 


\section{Introduction}

Supersymmetry is a powerful tool for extracting exact information about quantum field theories. Supersymmetry algebras that contain R-charges in the right hand side have special BPS multiplets. These multiplets occur at special values of energies or conformal dimensions determined by their charge, and have fewer states than the generic representation. An infinitesimal change in the energy of a special multiplet turns it into a generic multiplet with a discontinuously larger number of states. One might be tempted to use this observation to conclude that the number of short representations cannot change under variation of any continuous parameter of the field theory; however there is a caveat. It is sometimes possible for two or more BPS representations to combine into a generic representation. For this reason only states that cannot combine with other multiplets to form a long representation are guaranteed to be protected.

In this paper we construct some quantities, called indices, that receive contributions only from those BPS states that cannot combine into long representations. The indices that we construct are defined for 4 dimensional superconformal field theories (with arbitrary number of supersymmetries) on $S^{3} \times$ time. They take the form

$$
\mathcal{I}^{W}=\operatorname{Tr}\left[(-1)^{F} e^{\mu_{i} q_{i}}\right]
$$

where $q_{i}$ are charges that commute with a particular supercharge. Our indices closely resemble the Witten index [1], and are invariant under all continuous deformations of the theory that preserve superconformal invariancel. We demonstrate that our indices $\mathcal{I}^{W}$ contain all the information about protected states that can be obtained by group theory alone, and so should be useful in the study of general super-conformal field theories.

The indices $\mathcal{I}^{W}$ are a functions of 2,3 and 4 continuous variables for $\mathcal{N}=1,2,4$ superconformal field theories respectively. In the case of the $\mathcal{N}=4$ Yang Mills theory we explicitly compute this index $\mathcal{I}_{Y M}^{W}$ in the free limit. Upon taking the large $N$ limit the index receives contributions only from states with energies of order one at all chemical potentials of order one. In other words $\mathcal{I}_{Y M}^{W}$ does not undergo the deconfinement phase transition described in [2, 3]. Moreover we find that $\mathcal{I}_{Y M}^{W}$ agrees perfectly with the index evaluated over the spectrum of free ten dimensional massless fields propagating on $A d S_{5} \times S^{5}$. This

1 More generally they invariant under all deformations of the theory that preserve the corresponding supercharge. 
agreement provides a check on the AdS/CFT conjecture in the BPS sector, which ends up containing the same information as the matching of chiral primary operators in [4,5].

Related to the fact that the index never 'deconfines', in the limit of very small chemical potential, with charges growing like $q \sim N^{2}$ we find that the index $\mathcal{I}_{Y M}^{W}$ grows rather slowly. In particular, it does not grow fast enough to account for the entropy of the BPS black holes in $A d S_{5} \times S^{5}$ found in [6, [7,8]. This is not a contradiction with $A d S / C F T$; the entropy of a black hole counts all supersymmetric states with a positive sign whereas our index counts the same states up to sign. It is possible for cancellations to ensure that the Index is much smaller than the partition function evaluated over supersymmetric states of the theory. This is certainly what happens in the free $\mathcal{N}=4$ theory, where both quantities (the index and the partition function) may explicitly be computed, and is presumably also the case at strong coupling.

It may well be possible to provide a weak coupling microscopic counting of the entropy of BPS black holes [6, [7,8] in $A d S_{5} \times S^{5}$; however such an accounting must incorporate some dynamical information about $\mathcal{N}=4$ super Yang Mills beyond the information contained in the superconformal algebra. In this paper we make some small steps towards understanding the entropy of these black holes. In particular we provide a counting of the entropy for small black holes in terms of D-branes and giant gravitons in the interior. The counting is rather similar to the one performed for the D1D5p black holes [9]. We also note that, for large (large compared to the AdS radius) black holes a naive computation of the simple partition function of BPS states in the free theory gives a formula which has similar features to the black hole answer.

The indices (1.1) do not exhaust all interesting calculable information about supersymmetric states in all superconformal field theory; in specific examples it is possible to extract more refined information about supersymmetric states by adding extra input. An explicit example where dynamical information allows us to make more progress is the computation of the chiral ring [5,10]. In the case of $\mathcal{N}=4$ Yang Mills theory, we write down explicit counting formulas for $1 / 2,1 / 4$ and $1 / 8$ BPS states. The counting can be done in terms of $N$ particles in harmonic oscillator potentials. For very large charges the entropy in these states grows linearly in $N$. By taking the large $N$ limit of these partition functions we show that they display a second order phase transition which corresponds to the formation of Bose-Einstein condensate.

The structure of this paper is as follows. In section 2 we review the unitary representations of the conformal and superconformal algebra, and list the linear combinations 
of (numbers of) short representations that form indices in these algebras. In section 3 we define the Witten Indices that are the main topic of this paper, and explain how these are related to the indices of section 2. This comparison allows us to argue that our Indices capture all group theoretically protected information about short representations in superconformal field theories. In section 4 we turn to the $\mathcal{N}=4$ Super Yang Mills theory. We compute our index in this theory in free Yang Mills on $S^{3}$ and show that it agrees perfectly, in the large $N$ limit, with the same index computed over supergravitons in $A d S_{5} \times S^{5}$. In section 5 we continue our study of supersymmetric states in the $\mathcal{N}=4$ Yang Mills theory on $S^{3}$. We compute the partition function over $\frac{1}{16}^{\text {th }}$ supersymmetric states in free Yang Mills using perturbation theory and in strongly coupled Yang Mills using gravity, and compare the two results. In section 6 present exact formulas for the partition function

over $\frac{1}{4}^{\text {th }}$ and $\frac{1}{8}^{\text {th }}$ BPS states in $\mathcal{N}=4$ Yang Mills. For large charges we find that the free energy of these states scales linearly in $N$. This free energy displays a second order transition which is associated to the formation of a Bose-Einstein condensate.

This paper contains two related but distinct streams. Section 2 and 3 below concern themselves with the detailed nature of unitary representations of the superconformal algebra. Sections 4, 5 and 6 study the supersymmetric states of the $\mathcal{N}=4$ Yang Mills theory on $S^{3}$. The link between these two streams is the Witten index, defined in section 3.1. The reader who is interested only in the definition of the index and the results for $\mathcal{N}=4$ Yang Mills could proceed directly to section 3.1 where the index is defined, then to sections 4 , 5 and 6 for computations in the $\mathcal{N}=4$ Yang Mills theory. On the other hand, the reader who is interested principally in the algebraic aspects of this index, including the demonstration that the Witten index captures all protected information about superconformal field theories in four dimensions, could focus on sections two and three.

In this revised version of the paper we have added section 6.2 which shows, using the index, that a particular double trace operator in the $\mathbf{2 0}$ of $S O(6)$ is protected.

While this paper was being completed we saw [11] which overlaps with parts of section 3.

\section{4 dimensional Superconformal Algebras and their Unitary Representations}

In this section we study the structure of representations of conformal and superconformal algebras. Our goal is to understand which representations, or combinations of representations, are protected. This will allow us to show that all protected information 
that can be obtained by using group theory alone is captured by the index we will define in section 3.1. The reader who is willing to accept this fact (and is otherwise uninterested in the structure of unitary representations of the superconformal algebra), can just jump to section 3.1 and from there to section 4. We start this section with a discussion of the conformal algebra and then we discuss the superconformal algebra.

\subsection{The 4 dimensional Conformal Algebra}

The set of killing vectors $M_{\mu \nu}=-i\left(x_{\mu} \partial_{\nu}-x_{\nu} \partial_{\mu}\right), P_{\mu}=-i \partial_{\mu}, K_{\mu}=i\left(2 x_{\mu} x . \partial-x^{2} \partial_{\mu}\right)$ and $H=x . \partial$ form a basis for infinitesimal conformal diffeomorphisms of $R^{4}$. These killing vectors generate the algebra

$$
\begin{aligned}
{\left[H, P_{\mu}\right] } & =P_{\mu}, \\
{\left[H, K_{\mu}\right] } & =-K_{\mu}, \\
{\left[K_{\mu}, P_{\nu}\right] } & =2\left(\delta_{\mu \nu} H-i M_{\mu \nu}\right), \\
{\left[M_{\mu \nu}, P_{\rho}\right] } & =i\left(\delta_{\mu \rho} P_{\nu}-\delta_{\nu \rho} P_{\mu}\right), \\
{\left[M_{\mu \nu}, K_{\rho}\right] } & =i\left(\delta_{\mu \rho} K_{\nu}-\delta_{\nu \rho} K_{\mu}\right), \\
{\left[M_{\mu \nu}, M_{\rho \sigma}\right] } & =i\left(\delta_{\mu \rho} M_{\nu \sigma}+\delta_{\nu \sigma} M_{\mu \rho}-\delta_{\mu \sigma} M_{\nu \rho}-\delta_{\nu \rho} M_{\mu \sigma}\right) .
\end{aligned}
$$

Consider a 4 dimensional Euclidean quantum field theory. It is sometimes possible to combine the conformal killing symmetries of the previous paragraph with suitable action on fields to generate a symmetry of the theory. In such cases the theory in question is called a conformal field theory (CFT). The Euclidean path integral of a CFT may be given a useful Hilbert space interpretation via a radial quantization. Wave functions (kets) are identified with the path integral, with appropriate operator insertions, over the unit 3 ball surrounding the origin. Dual wave functions (bras) are obtained by acting on kets with by the conformal symmetry corresponding to inversions $x^{\mu}=\frac{x^{\mu}}{x^{2}}$ 2. Under an inversion, the killing vectors of the previous paragraph transform as $M_{\mu \nu} \rightarrow M_{\mu \nu}, H \rightarrow-H, P_{\mu} \rightarrow K_{\mu}$. As a consequence, the operators $M_{\mu \nu}, P_{\mu}, K_{\nu}$ are represented on the CFT Hilbert space (2.1) with the hermiticity conditions

$$
M_{\mu \nu}=M_{\mu \nu}^{\dagger}, \quad P_{\mu}=K_{\mu}^{\dagger}
$$

2 As a consequence, a bra may be thought of as being generated by a path integral, performed with appropriate insertions, on $R^{4}$ minus the unit 3 ball. The scalar product between a bra and a ket is the path integral - with insertions both inside and outside the unit sphere - over all of space. 
Radial quantization of the CFT on $R^{4}$ is equivalent to studying the field theory on $S^{3} \times$ time. The operators $M_{\mu \nu}$ generate the $S O(4)$ rotational symmetries of $S^{3}$, and $H$ is the Hamiltonian. From this point of view the conjugate generators $P_{\mu}$ and $K_{\mu}$ are less familiar; they act as ladder operators, respectively raising and lowering energy by a single unit.

The Hilbert space of a CFT on $S^{3} \times$ time may be decomposed into a sum of irreducible unitary representations of the conformal group. The theory of these representations was studied in detail by [12]. We present a brief review below, as a warm up for the superconformal algebra (see [13] and references therein for a recent discussion).

\subsection{Unitary Representations of the Conformal Group}

Any irreducible representation of the conformal group can be written as some direct sum of representations, $R_{\text {compact }}^{i}$, of the compact subgroup $S O(4) \times S O(2)$ :

$$
R_{S O(4,2)}=\sum_{i} \bigoplus R_{\text {compact }}^{i}
$$

The states within a given $S O(4) \times S O(2)$ representation all have the same energy. As the energy spectrum of any sensible quantum field theory is bounded from below, the representations of interest to us all possess a particular set of states with minimum energy. We will call these states (which we will take to transform as $R_{\text {compact }}^{\lambda}$ ) the lowest weight states. The $K^{\mu}$ operators necessarily annihilate all the states in $R_{\text {compact }}^{\lambda}$ because the $K^{\mu}$ have negative energy. We can now act on these lowest weight states with an arbitrary number of $P_{\mu}$ ('raising') operators to generate the remaining states in the representation. We will use the charges of the lowest weight state $|\lambda\rangle \equiv\left|E, j_{1}, j_{2}\right\rangle$ to label this representation. We use the fact that $S O(4)=S U(2) \times S U(2) ; j_{1}$ and $j_{2}$ are standard representation labels of these $S U(2) s$.

It is important that not all values of $E, j_{1}, j_{2}$ yield unitary representations of the conformal group. For a representation to be unitary, it is necessary for all states to have positive norm. Acting on the lowest energy states with $P_{\mu}$, we obtain (via a Clebsh Gordan decomposition) states that transform in the representations $\left(E+1, j_{1} \pm 1 / 2, j_{2} \pm 1 / 2\right)$. The norm of these states may be calculated using the commutation relations (2.1) [14]. The states with lowest norm turn out to have quantum numbers $\left(E+1, j_{1}-\frac{1}{2}, j_{2}-\frac{1}{2}\right)$, and this norm is given by

$$
\frac{\|\|^{2}}{2}=E-j_{1}-1+\delta_{j_{1} 0}-j_{2}-1+\delta_{j_{2} 0} .
$$


Unitarity then requires that

$$
\begin{array}{ll}
\text { (i) } \quad E \geq j_{1}+j_{2}+2 & j_{1} \neq 0 \quad j_{2} \neq 0 \\
\text { (ii) } \quad E \geq j_{1}+j_{2}+1 & j_{1} j_{2}=0 .
\end{array}
$$

The special case $j_{1}=j_{2}=0$ has an additional complication. In this case the norm of the level 2 state $P^{2}|\psi\rangle$ is proportional [14] to $E(E-1)$ and so is negative for $0<E<1$. The representation with $E=0$ is annihilated by all momentum operators and represents the vacuum state. The representation at $E=1$ is short and it obeys the equation $P^{2}|E\rangle=0$ so it is a free field in the conformal field theory.

Unitary representations exist even when this bound is strictly saturated. The zero norm states, and all their descendants, are simply set to zero in these representations 3 making them shorter than generic.

Now consider a one parameter (fixed line) of conformal field theories. An infinitesimal variation of the parameter that labels the theory will, generically, result in an infinitesimal variation in the energy of all the long representations of the theory. However a short representation can change its energy only if it turns into a long representation. In order for this to happen without a discontinuous jump in the spectrum of the CFT (i.e. a phase transition), it must pair up with some other representation, to make up the states of a long representation with energy at just above the unitarity threshold. Groups of short representations that can pair up in this manner are not protected; the numbers of such representations can jump discontinuously under infinitesimal variations of a theory.

However consider an index $I$ that is defined as a sum of the form

$$
I=\alpha[i] n[i]
$$

where $i$ runs over the various short representations of the theory, $n[i] s$ are the number of representations of the $i^{t h}$ variety, and $\alpha[i]$ are fixed numbers chosen so that $I$ evaluates to zero on any collection of short representations that can pair up into long representations. By definition such an index is unaffected by groups of short representations pairing up and leaving, as it evaluates to zero anyway on any set of representations that can. It follows that an index cannot change under continuous deformations of the theory.

3 The consistency of this procedure relies on the fact that, at the unitarity bound, zero norm states are orthogonal to all states in the representation. As a consequence the inner product on the representation modded out by zero norm states is well defined and positive definite. 
We will now argue that the conformal algebra does not admit any non trivial indices. In order to do this we first list how a long representation decomposes into a sum of other representations (at least one of which is short) when its energy is decreased so that it hits the unitarity bound. Let us denote the representations as follows. $A_{E, j_{1}, j_{2}}$ denotes the generic long representation, $C_{j_{1}, j_{2}}$ denotes the short representations with $j_{1}$ and $j_{2}$ both not equal to zero, $B_{j_{1}}^{L}$ denotes the short representations with $j_{2}=0, B_{j_{2}}^{R}$ the short ones with $j_{1}=0$. Finally we denote the special short representation at $E=1$ and $j_{1}=j_{2}=0$ by $B$. As the energy is decreased to approach the unitarity bound we find

$$
\begin{aligned}
& \lim _{\epsilon \rightarrow 0} \chi\left[A_{j_{1}+j_{2}+2+\epsilon, j_{1}, j_{2}}\right]=\chi\left[C_{j_{1}, j_{2}}\right]+\chi\left[A_{j_{1}+j_{2}+3, j_{1}-\frac{1}{2}, j_{2}-\frac{1}{2}}\right] \\
& \lim _{\epsilon \rightarrow 0} \chi\left[A_{j_{1}+1+\epsilon, j_{1}, 0}\right]=\chi\left[B_{j_{1}}^{L}\right]+\chi\left[C_{j_{1}-\frac{1}{2}, \frac{1}{2}}\right] \\
& \lim _{\epsilon \rightarrow 0} \chi\left[A_{j_{2}+1+\epsilon, 0, j_{2}}\right]=\chi\left[B_{j_{2}}^{R}\right]+\chi\left[C_{\frac{1}{2}, j_{2}-\frac{1}{2}}\right] \\
& \lim _{\epsilon \rightarrow 0} \chi\left[A_{1+\epsilon, 0,0}\right]=\chi[B]+\chi\left[A_{3,0,0}\right] .
\end{aligned}
$$

In (2.7) and throughout this paper, the symbol $\chi$ denotes the super-character on a representationt.

It follows from (2.7) that $\sum_{i} \alpha_{i} n_{i}$ is an index only if

$$
\alpha_{C_{j_{1}, j_{2}}}=0, \quad \alpha_{B_{j_{1}}^{L}}+\alpha_{C_{j_{1}-\frac{1}{2}, \frac{1}{2}}}=0, \quad \alpha_{B_{j_{2}}^{R}}+\alpha_{C_{\frac{1}{2}, j_{2}-\frac{1}{2}}}=0, \quad \alpha_{B}=0 .
$$

The only solution to these equations has all $\alpha$ to zero; consequently the conformal algebra admits no nontrivial indices. The superconformal algebra will turn out to be more interesting in this respect.

\subsection{Unitary Representations of $d=4$ Superconformal Algebras}

In the next two subsections we review the unitary representations of the $d=4 \mathrm{su}-$ perconformal algebras [15] that were studied in [16, 17, 18, 19, 14, 20, 21]. A supersymmetric field theory that is also conformally invariant, actually enjoys superconformal symmetry, a symmetry algebra that is larger than the union of conformal and super symmetry algebras. The bosonic subalgebra of the $\mathcal{N}=m$ superconformal algebra consists of the conformal algebra times $U(m)$, except in the special case $m=4$, where the $R$ symmetry algebra is

4 i.e. $\operatorname{Tr}_{R}(-1)^{F} G$ where $R$ is an arbitrary representation, $G$ is an arbitrary group element, and $F$ is the Fermion number, which plays no role in the representation theory of the conformal group, but will be important when we turn superconformal groups below. 
$S U(4)$. The fermionic generators of this algebra consist of the $4 m$ supersymmetry generators $Q^{\alpha i}$ and $\bar{Q}_{i}^{\dot{\alpha}}$, together with the super conformal generators $S_{\alpha i}, \bar{S}_{\dot{\alpha}}^{j}$. The generators transform under $S O(4) \times U(m)$ as indicated by their index structure (an upper $i$ index indicates a $U(m)$ fundamental, while a lower $i$ index is a $U(m)$ anti-fundamental). The commutation relations of the algebra are listed in detail in Appendix A.1. In particular,

$$
\left\{S_{\alpha i}, Q^{\beta j}\right\}=\delta_{i}^{j}\left(J_{1}\right)_{\alpha}^{\beta}+\delta_{\alpha}^{\beta} R_{i}^{j}+\delta_{i}^{j} \delta_{\alpha}^{\beta}\left(\frac{H}{2}+r \frac{4-m}{4 m}\right)
$$

where $\left(J_{1}\right)_{\alpha}^{\beta}$ are the $S U(2)$ generators in spinor notation, $R_{i}^{j}$ are the $S U(m)$ generators and $r$ is the $U(1)$ generator. As in the previous subsection, radial quantization endows these generators with hermiticity properties; specifically

$$
\left(Q^{\alpha i}\right)^{\dagger}=S_{\alpha i}, \quad\left(\bar{Q}_{i}^{\dot{\alpha}}\right)^{\dagger}=\bar{S}_{\dot{\alpha}}^{i}
$$

The theory of unitary representations of the superconformal algebra is similar to that of the conformal algebra. Irreducible representations are labeled by the energy $E$ and the $S U(2) \times S U(2)$ and $U(m)$ representations of their lowest weight states. We label $U(m)$ representations by their $U(1)$ charge $r$ (normalized such that $Q^{\alpha i}$ has charge +1 and $\bar{Q}_{i}^{\dot{\alpha}}$ has charge -1) and the integers $R_{k}(k=1 \ldots m-1)$, the number of columns of height $k$ in the Young Tableaux for the representation.

Lowest weight states are annihilated by the $S$ but, generically, not by the $Q$ operators. $Q^{\alpha i}$ have $E=\frac{1}{2}$ and transform in the $S U(2) \times S U(2) \times U(m)$ representation with quantum numbers $j_{1}=\frac{1}{2}, \quad j_{2}=0, \quad r=1, \quad R_{1}=1, \quad R_{i}=0 \quad(i>1)$. Let $\left|\psi_{a}\right\rangle$ be the set of lowest weight states of this algebra that transforms in the representation $\left(E, j_{1}, j_{2}, r, R_{i}\right)$. The states $Q^{\alpha i}\left|\psi_{a}\right\rangle$ transform in all the Clebsh Gordan product representations; the lowest norm among these states occurs for those that have quantum numbers $\left(E+\frac{1}{2}, j_{1}-\frac{1}{2}, j_{2}, r+\right.$ $\left.1, R_{1}+1, R_{j}\right)$; the value of the norm of these states is given by [14]

$$
\begin{aligned}
2\left\|\chi_{1}\right\|^{2} & =E+2 \delta_{j_{1}, 0}-E_{1}\left(j_{1}, r, R_{i}\right), \\
E_{1} & \equiv 2+2 j_{1}+2 \frac{\sum_{p=1}^{m-1}(m-p) R_{p}}{m}+\frac{r(4-m)}{2 m} .
\end{aligned}
$$

$5 R_{k}$ may also be thought of as the eigenvalues of the highest weight vectors under the diagonal generator $R_{k}$ whose $k^{t h}$ diagonal entry is unity, $(k+1)^{t h}$ entry is -1 , and all other are zero, in the defining representation. 
In a similar fashion, of the states of the form $\bar{Q}_{\dot{\alpha} i}|\psi\rangle$ the lowest norm occurs for those that transform in $\left(E+\frac{1}{2}, j_{1}, j_{2}-\frac{1}{2}, r-1, R_{k}, R_{m-1}+1\right)$, and the norm of these states is equal to 14

$$
\begin{aligned}
2\left\|\chi_{2}\right\|^{2} & =E+2 \delta_{j_{2}, 0}-E_{2}\left(j_{1}, j_{2}, r, R_{i}\right) \\
E_{2} & \equiv 2+2 j_{2}+\frac{2 \sum_{p=1}^{m-1} p R_{p}}{m}-\frac{r(4-m)}{2 m} .
\end{aligned}
$$

Clearly unitarity demands that $\left\|\chi_{1}\right\|^{2} \geq 0$ and $\left\|\chi_{2}\right\|^{2} \geq 0$. As for the conformal group, representations with either $\left\|\chi_{1}\right\|^{2}=0$ or $\left\|\chi_{2}\right\|^{2}=0$ or both zero are allowed. In such representations the zero norm states and all their descendants are simply set to zero, yielding short representations.

In the special case $j_{1}=0$ the positivity of the norm at level 2 yields more information. Of states of the form $Q^{\alpha i} Q^{\beta j}\left|\psi_{a}\right\rangle$ (where $\left|\psi_{a}\right\rangle$ are the lowest weight states), those that have the smallest norm transform in the representation $\left(E+1,0, j_{2}, r+2, R_{1}+2, R_{j}\right)$. The norm of these states turns out to be proportional to $\left(E-E_{1}\right)\left(E-E_{1}+2\right)$ where $E_{1}$ is defined in (2.11). Thus unitarity disallows representations in the window $E_{1}-$ $2<E<E_{1}$. Representations at $E=E_{1}-2$ and $E=E_{1}$ are both short and both allowed. Representations at $E=E_{1}-2$ are special because they are separated from long representations (with the same value of all other charges) by an energy gap of two units. All these remarks also apply to the special case $j_{2}=0$, upon replacing $Q^{\alpha i}$ with $\bar{Q}_{i}^{\dot{\alpha}}$ and $E_{1}$ with $E_{2}$.

In [20], Dolan and Osborn, performed a comprehensive tabulation of short representations of the $d=4$ superconformal algebras. We will adopt a notation that is slightly different from theirs. Representations are denoted by $\mathbf{x}^{\mathbf{L}} \mathbf{x}_{E, j_{1}, j_{2}, r, R_{i}}$ where

$$
\mathbf{x}^{\mathbf{L}}= \begin{cases}\mathbf{a} & \text { if } E>E_{1} \\ \mathbf{c} & \text { if } E=E_{1} \text { and } j_{1} \geq 0 \\ \mathbf{b} & \text { if } E=E_{1}-2 \text { and } j_{1}=0\end{cases}
$$

and

$$
\mathbf{x}^{\mathbf{R}}= \begin{cases}\mathbf{a} & \text { if } E>E_{2} \\ \mathbf{c} & \text { if } E=E_{2} \text { and } j_{2} \geq 0 \\ \mathbf{b} & \text { if } E=E_{2}-2 \text { and } j_{2}=0\end{cases}
$$

Further, we will usually omit to specify the first (energy) subscript on all short representations as this energy is determined by the other charges. Thus representations denoted by aa are long; all other representations are short. 


\subsection{The Null Vectors in Short Representations}

We now study the nature of the null vectors in short representations in more detail. Consider a representation of the type $\mathbf{c x}$, with $j_{1}>0$, where $\mathbf{x}$ is either $\mathbf{a}, \mathbf{c}$ or b. Such a representation has $\left\|\chi_{1}\right\|^{2}=0$. The descendants of the null-state form another representation of the superconformal algebra. This representation also has null states 6 characterized by their own value of $\left(\left\|\chi_{1}^{\prime}\right\|^{2},\left\|\chi_{2}^{\prime}\right\|^{2}\right)$. A short calculation $\nabla_{\text {shows }}$ that $\left.\left\|\chi_{1}^{\prime}\right\|^{2},\left\|\chi_{2}^{\prime}\right\|^{2}\right) /=\left(0,\left\|\chi_{2}\right\|^{2}\right)$. It follows that the $Q$ null states of a representation of type $\mathbf{c x}$ are generically also of the type $\mathbf{c x}$. The exception to this rule occurs when $j_{1}=0$, in which case the null states are of type bx. Of course analogous statements are also true for $\bar{Q}$ null states. All of this may be summarized in a set of decomposition formulae, for the supercharacters,

$$
\chi[R]=\operatorname{Tr}_{R}\left[(-1)^{2\left(J_{1}+J_{2}\right)} G\right],
$$

where $G$ is an arbitrary element of the superconformal group. These formulae describe how a long representation decomposes into a set of short representation when its energy hits the unitarity bound.

$$
\begin{aligned}
& \lim _{\epsilon \rightarrow 0} \chi\left[\mathbf{a a}_{E_{1}+\epsilon, j_{1}, j_{2}, r, R_{i}}\right]=\chi\left[\widetilde{\mathbf{c}} \mathbf{a}_{j_{1}, j_{2}, r, R_{i}}\right]+\chi\left[\widetilde{\mathbf{c}} \mathbf{a}_{j_{1}-\frac{1}{2}, j_{2}, r+1, R_{1}+1, R_{j}}\right], E_{1}>E_{2} \\
& \lim _{\epsilon \rightarrow 0} \chi\left[\mathbf{a a}_{E_{2}+\epsilon, j_{1}, j_{2}, r, R_{i}}\right]=\chi\left[\mathbf{a}_{j_{1}, j_{2}, r, R_{i}}\right]+\chi\left[\mathbf{a}_{j_{1}, j_{2}-\frac{1}{2}, r-1, R_{k}, R_{m-1}+1}\right], E_{2}>E_{1} \\
& \lim _{\epsilon \rightarrow 0} \chi\left[\mathbf{a a}_{E_{2}+\epsilon, j_{1}, j_{2}, r, R_{i}}\right]=\chi\left[\widetilde{\mathbf{c}}_{j_{1}, j_{2}, r, R_{i}}\right]+\chi\left[\widetilde{\mathbf{c}}_{j_{1}-\frac{1}{2}, j_{2}, r+1, R_{1}+1, R_{j}}\right]+ \\
& \chi\left[\widetilde{\mathbf{c}}_{j_{1}, j_{2}-\frac{1}{2}, r-1, R_{k}, R_{m-1}+1}\right]+\chi\left[\widetilde{\mathbf{c}}_{j_{1}-\frac{1}{2}, j_{2}-\frac{1}{2}, r, R_{1}+1, R_{l}, R_{m-1}+1}\right], E_{1}=E_{2}
\end{aligned}
$$

where, in this equation and, as far as possible, in the rest of the paper, we use the index convention

$$
i=1 \ldots m-1, \quad j=2 \ldots m-1, \quad k=1 \ldots m-2, \quad l=2 \ldots m-2 .
$$

6 When we say that a short representation has 'null states' of a particular type we mean the following. When we lower the energy of a long representation down to its unitarity bound $\left(E_{1}\right.$ or $E_{2}$ ), the long representation splits into a positive norm short representation $m$, plus a set of null representations $m^{\prime}$. We describe this situation by the words 'the short representation $m$ has null representations $m^{\prime}$. As is clear from this definition, it is meaningless to talk of the null state content of representations of the sort $\mathbf{b x}$ or $\mathbf{x b}$, as these representations are separated by a gap from long representations.

$$
{ }^{7}\left(\left\|\chi_{1}^{\prime}\right\|^{2},\left\|\chi_{2}^{\prime}\right\|^{2}\right)=\left(\frac{1}{2}+1-2(m-1) / m-(4-m) / 2 m,\left\|\chi_{2}\right\|^{2}-\frac{1}{2}+2 / m+(4-m) / 2 m\right)=\left(0,\left\|\chi_{2}\right\|^{2}\right) .
$$


On the right hand side of (2.16) we have used the notation given in table 1.

Table 1 : Short Representations

\begin{tabular}{|c|c|}
\hline Symbol & Denotation \\
\hline$\widetilde{\mathbf{c}} \mathbf{a}_{j_{1}, j_{2}, r, R_{i}}$ & $\begin{array}{c}\mathbf{c a}_{j_{1}, j_{2}, r, R_{i}}, \quad j_{1} \geq 0 \\
\mathbf{b a}_{0, j_{2}, r+1, R_{1}+1, R_{j}, \quad j_{1}=-\frac{1}{2}}\end{array}$ \\
\hline $\mathbf{a} \widetilde{\mathbf{c}}_{j_{1}, j_{2}, r, R_{i}}$ & $\begin{array}{c}\mathbf{a c}_{j_{1}, j_{2}, r, R_{i}}, \quad j_{2} \geq 0 \\
\mathbf{a b}_{j_{1}, 0, r-1, R_{k}, R_{m-1}+1}, \quad j_{2}=-\frac{1}{2}\end{array}$ \\
\hline$\widetilde{\mathbf{c}}_{j_{1}, j_{2}, r, R_{i}}$ & 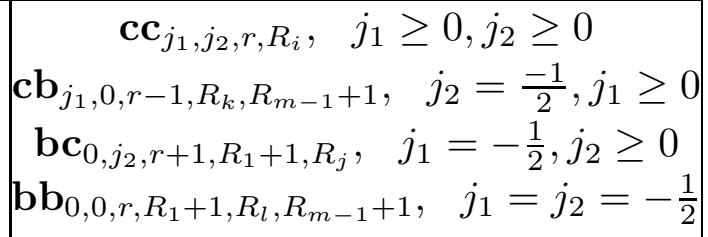 \\
\hline
\end{tabular}

\subsection{Indices For Four Dimensional Super Conformal Algebras}

We now turn to a study of the indices for these algebras. Using (2.16) it is not difficult to convince oneself that the set of Indices for the four dimensional superconformal field theories is a vector space that is spanned by

1. The number of representations of the sort bx with $R_{1}=0$ or $R_{1}=1$ plus the number of representations of the sort $\mathbf{x b}$ with $R_{m-1}=0$ or $R_{m-1}=1$.

2. The Indices

$$
I_{j_{2}, \widehat{r}, M, R_{j}}^{L}=\sum_{p=-1}^{M}(-1)^{p+1} n\left[\widetilde{\mathbf{c}} \mathbf{x}_{\frac{p}{2}, j_{2}, \widehat{r}-p, M-p, R_{j}}\right]
$$

for all values of $\widehat{r}$ and non negative integral values of $j_{2}, M, R_{j}$. In the case $m=1$ we do not have the indices $M$ or $R_{j}$ and the sum runs from $p=-1$ to infinity. In the $m=4$ case, simply ignore the $r$ and $\widehat{r}$ subindices.

3. The Indices

$$
I_{j_{1}, r^{\prime \prime}, R_{k}, N}^{R}=\sum_{p=-1}^{M}(-1)^{p+1} n\left[\mathbf{x}_{j_{1}, \frac{p}{2}, r^{\prime \prime}+p, R_{k}, N-p}\right]
$$

for all values of $r^{\prime \prime}$ and non negative integral values of of $j_{1}, R_{k}, N$, with the same remarks for $m=1,4$.

In the special case that representations that contribute to the sum in (2.18) and (2.19) have quantum numbers on which $E_{1}=E_{2}$, the indices (2.18) and (2.19) are subject to

8 If this relation is true for any term that contributes to the sum, it is automatically true on all other terms as well. 
the additional constraints

$$
\sum_{p=-1}^{N}(-1)^{p} I_{\frac{p}{2}, r^{\prime \prime \prime}+p, M, R_{l}, N-p}^{L}=\sum_{p=-1}^{M}(-1)^{p} I_{\frac{p}{2}, r^{\prime \prime \prime}-p, M-p, R_{l}, N}^{R} \quad\left(E_{1}=E_{2}\right)
$$

for all values of $r^{\prime \prime \prime}=-\infty \ldots \infty$, and non negative integral values of $M, N, R_{l}$.

These results are explained in more detail in Appendix B.1, where we also present a detailed listing of the absolutely protected multiplets, for the $\mathcal{N}=1,2,4$ superconformal algebras.

\section{A Trace Formula for the Indices of Superconformal Algebra}

The supercharges $Q^{\alpha i}$ transform in the fundamental or $(1,0, \ldots, 0)$ representation of $S U(m)$. Let $Q \equiv Q^{-\frac{1}{2}, 1}$, the supercharge whose $S U(2) \times S U(2)$ quantum numbers are $\left(j_{1}^{3}, j_{2}^{3}\right)=\left(-\frac{1}{2}, 0\right)$, that has $r=1$, and whose $S U(m)$ quantum numbers are $(1,0, \ldots 0)$. Let $S \equiv Q^{\dagger}$. Then (see (2.9))

$$
2\{S, Q\}=H-2 J_{1}-2 \sum_{k=1}^{m-1} \frac{m-k}{m} R_{k}-\frac{(4-m) r}{2 m}=E-\left(E_{1}-2\right) \equiv \Delta .
$$

It follows from (3.1) that every state in a unitary representation of the superconformal group has $\Delta \geq 0$. Note that the Jacobi identity implies that $Q$ and $S$ commute with $\Delta$.

Consider a unitary representation $R$ of the superconformal group that is not necessarily irreducible. Let $R_{\Delta_{0}}$ denote the linear vector space of states with $\Delta=\Delta_{0}>0$. It follows from (3.1) that if $|\psi\rangle$ is in $R_{\Delta_{0}}$ then

$$
|\psi\rangle=Q \frac{S}{\Delta_{0}}|\psi\rangle+S \frac{Q}{\Delta_{0}}|\psi\rangle .
$$

Let $R_{\Delta_{0}}^{Q}$ denote the subspace of $R_{\Delta_{0}}$ consisting of states annihilated by $Q$ and $R_{\Delta_{0}}^{S}$ the set of states in $R_{\Delta_{0}}$ that are annihilated by $S$. It follows immediately from (3.2) (and the unitarity of the representation) that $R_{\Delta_{0}}=R_{\Delta_{0}}^{Q}+R_{\Delta_{0}}^{S}$ and that $S|\psi\rangle=\left|\psi^{\prime}\right\rangle$ is a one to one map from $R_{\Delta_{0}}^{Q}$ to $R_{\Delta_{0}}^{S}\left(Q / \Delta_{0}\right.$ provides the inverse map).

Now consider the Witten index

$$
\mathcal{I}^{W L}=\operatorname{Tr}_{R}\left[(-1)^{F} \exp (-\beta \Delta+M)\right]
$$

where $M$ is any element of the subalgebra of the superconformal algebra that commutes with $Q$ and $S$. We discuss this subalgebra in detail in the next subsection. It follows that 
the states in $R_{\Delta_{0}}$ do not contribute to this index, the contribution of $R_{\Delta_{0}}^{Q}$ cancels against that of $R_{\Delta_{0}}^{S}$. Consequently, $\mathcal{I}^{W L}$ receives contributions only from states with $\Delta=0$, i.e. those states that are annihilated by both $Q$ and $S$. Thus, despite appearances, (3.3) is independent of $\beta$. As no long representation contains states with $\Delta=0$, such representations do not contribute to $\mathcal{I}^{W L}$. It also follows from continuity that $\mathcal{I}^{W L}$ evaluates to zero on groups of short representations that a long representation breaks up into when it hits unitarity threshold. As a consequence $\mathcal{I}^{W L}$ is an index; it cannot change under continuous variations of the superconformal theory, and must depend linearly on the Indices, $I^{L}$ and $I^{R}$, listed in the previous section. We will explain the relationship between $\mathcal{I}^{W L}$ and $I^{L}$ in more detail in subsection 3.2 and 3.3 below. The main result of the following subsections is to show that (3.3) (and its $\mathcal{I}^{W R}$ version) completely capture the information contained in the indices defined in the previous section, which is all the information about protected representations that can be obtained without invoking any dynamical assumption. In appendix B.2 we derive most of the results of section 2 in a way that uses a smaller amount of group theory.

\subsection{The Commuting Subalgebra}

In this subsection we briefly describe the subalgebra of the superconformal algebra that commutes with the $S U(1 \mid 1)$ algebra spanned by $Q, S, \Delta$.

The $\mathcal{N}=m, d=4$ superconformal algebra is the super-matrix algebra $S U(2,2 \mid m) 9$. Supersymmetry generators transform as bifundamentals under the bosonic subgroups $S U(2,2)$ and $S U(m)$. It is not difficult to convince oneself that the commuting subalgebra we are interested in is $S U(2,1 \mid m-1) 10$. The generators of $S U(2,1 \mid m-1)$ are related to those of $S U(2,2 \mid \mathrm{m})$ via the obvious reduction. In more detail, the bosonic subgroup of $S U(2,1 \mid m-1)$ is $S U(2,1) \times U(m-1)$. The $U(m-1)$ factor sits inside the superconformal $U(m)$ setting all elements the first row and first column to zero, except for the 11 element which is set to one. The Cartan elements $\left(E^{\prime}, j_{2}^{\prime}, r^{\prime}, R_{i}^{\prime}\right)$ of the subalgebra are given in terms of those for the full algebra by

$$
E^{\prime}=E+j_{1}, \quad j_{2}^{\prime}=j_{2}, \quad r^{\prime}=\frac{(m-1) r}{m}-\sum_{p=1}^{m-1} \frac{m-p}{m} R_{p}, \quad R_{k}^{\prime}=R_{k+1}
$$

\footnotetext{
9 For $m=4$ we have $\operatorname{PSU}(2,2 \mid 4)$.

10 Or $P S U(2,1 \mid 3)$ for $m=4$.
} 
where $R_{k}^{\prime}$ are the Cartan elements of $U(m-1)$ and $r^{\prime}$ is the $U(1)$ charge in $U(m-1)$. We will think of (3.4) as defining a (many to one) map from $\left(E, j_{1}, j_{2}, r, R_{i}\right)$ to $\left(E^{\prime}, j_{2}^{\prime}, r^{\prime}, R_{i}^{\prime}\right)$

We will be interested in the representations of the subalgebra, $S U(2,1 \mid m-1)$, that are obtained by restricting a representation of the full algebra, $S U(2,2 \mid m)$, to states with $\Delta=0$. Null vectors, if any, of $S U(2,1 \mid m-1)$ are inherited from those of $S U(2,2 \mid m)$. Is is possible to show that $S U(2,1 \mid m-1)$ is short only when $S U(2,2 \mid m)$ is one of the representations $c b, c c$ or if $R$ is $b x$ with $R_{1}=0$. See Appendix B.3 for further discussion.

\section{2. $\mathcal{I}^{W L}$ expanded in sub-algebra characters with $I^{L}$ as coefficients}

In this subsection we present a formula for Index $\mathcal{I}^{W L}$ as a sum over super characters of the commuting subalgebra, $S U(2,1 \mid m-1)$, more details may be found in Appendix B.2.

It is not difficult to convince oneself (see Appendix B.2) that on any short irreducible representation $R$ of the superconformal algebra $S U(2,2 \mid m), \mathcal{I}^{W L}$ evaluates to the supercharacter of a single irreducible representation $R^{\prime}$ of the subalgebra $S U(2,1 \mid m-1)$. More specifically we find

$$
\begin{aligned}
\mathcal{I}^{W L}\left[\mathbf{b x}_{0, j_{2}, r, R_{i}}\right] & =\chi_{s u b}[\vec{b}] \\
\mathcal{I}^{W L}\left[\mathbf{c x}_{j_{1}, j_{2}, r, R_{i}}\right] & =(-1)^{2 j_{1}+1} \chi_{s u b}[\vec{c}]
\end{aligned}
$$

where $\chi_{\text {sub }}$ is the supercharacter

$$
\chi_{s}\left[R^{\prime}\right]=\operatorname{Tr}_{R^{\prime}}\left[(-1)^{2 J_{2}} G^{\prime}\right]
$$

where $G^{\prime}$ is an element of the Cartan subgroup. The vectors $\vec{b}$ and $\vec{c}$ specify the highest weight of the representation of the subalgebra in the Cartan basis $\left[E^{\prime}, j_{2}^{\prime}, r^{\prime}, R_{k}^{\prime}\right]$ defined in (3.4).

$$
\begin{aligned}
\vec{b} & =\left[\frac{3}{2} r-2 r^{\prime}, j_{2}, r^{\prime}, R_{j}\right], \\
\vec{c} & =\left[3+3\left(j_{1}+r / 2\right)-2 r^{\prime}, j_{2}, r^{\prime}, R_{j}\right]
\end{aligned}
$$

where $r^{\prime}$ is the function defined in (3.4); we emphasize the fact that it depends on $r$ and $R_{1}$ only through the combination $r-R_{1}$.

Notice that the functions that specify the character of the subalgebra, (3.7), are not

one to one. In fact, it follows from (3.5), (3.7), that $\mathcal{I}^{W L}$ evaluates to the same subalgebra character for each representation $R$ that appears in the sum in (2.18), for fixed values of $j_{2}, r^{\prime}, M, R_{i}$. Notice that by formally setting $j_{1}=-1 / 2$ in the second line of (3.7) we get the Cartan values for the subalgebra that we expect for the representation $\mathbf{b}$ according 
to the definition of $\widetilde{\mathbf{c}}$ in table 1 . This implies that we can replace $\mathbf{c}$ in (3.5) by $\widetilde{\mathbf{c}}$. More specifically

$$
\mathcal{I}^{W L}\left[\widetilde{\mathbf{c}}_{\frac{p}{2}, j_{2}, \widehat{r}-p, M-p, R_{j}}\right]=(-1)^{p} \mathcal{I}^{W L}\left[\widetilde{\mathbf{c}}_{0, j_{2}, \widehat{r}, M, R_{j}}\right]
$$

It follows immediately from (3.5), (3.8), that $\mathcal{I}^{W L}$, evaluated on any (in general reducible representation) $A$ of the superconformal algebra evaluates to

$$
\begin{aligned}
\mathcal{I}^{W L}[A]= & \sum_{j_{2}, r, R_{i}}\left(n\left[\mathbf{b x}_{0, j_{2}, r, 0, R_{i}}\right] \chi_{s u b}\left[\vec{b}_{0}\right]+n\left[\mathbf{b x}_{0, j_{2}, r, 1, R_{i}}\right] \chi_{s u b}\left[\vec{b}_{1}\right]\right) \\
& +\sum_{j_{2}, r^{\prime}, M, R_{i}} I_{j_{2}, \widehat{r}, M, R_{i}}^{L} \chi_{s u b}\left[\vec{c}_{0}\right]
\end{aligned}
$$

Where $\vec{b}_{0,1}$ are given by (3.7) with $R_{1}=0,1$ respectively and $\vec{c}_{0}$ is given by (3.7) with $j_{1}=0, r=\widehat{r}, R_{1}=M$. The quantities $n\left[\mathbf{x x}_{j_{1}, j_{2}, r, R_{i}}\right]$ in (3.9) are the number of copies of the irreducible representation, with listed quantum numbers, that appear in $A$, and $I_{j_{2}, \widehat{r}, M, R_{i}}^{L}$ are the indices (2.18) made out of these numbers.

Notice that most of the discussion in this section goes through unchanged if we were to consider the supergroup $S U(2 \mid 4)$ (or $S U(2 \mid m)$ ). The representation theory of this group was studied in [22,23] and the index was used in [24] to analyze various field theories with this symmetry. The index for the plane wave matrix model is given by an expression like (4.3) below but without the denominators (this is then inserted into (4.1)). Notice that the fact that the index for $N=4$ Yang Mills and the index for the plane wave matrix model are different implies that we cannot continuously interpolate between $\mathcal{N}=4$ super Yang Mills and the plane wave matrix model while preserving the $S U(2 \mid 4)$ symmetry. In 25] BPS representations and an index for $S U(1 \mid 4)$ were considered.

\subsection{The Witten Index $\mathcal{I}^{W R}$}

As in Section 2, we may define a second index $\mathcal{I}^{W R}$. The theory for this index is almost identical. We focus on the supercharge, $\bar{Q}_{m-1}^{-\frac{1}{2}}$ which has $S U(2) \times S U(2)$ quantum numbers, $\left(j_{1}^{3}, j_{2}^{3}\right)=\left(0,-\frac{1}{2}\right), r=-1$ and $S U(4)$ quantum numbers $(0,0, \ldots, 1)$. Let $\bar{S}=\bar{Q}^{\dagger}$.

It is then easy to verify (see Appendix A) that

$$
2\{\bar{S}, \bar{Q}\}=H-2 J_{2}-2 \sum_{k=1}^{m-1} \frac{k}{m} R_{k}+\frac{(4-m) r}{2 m}=E-\left(E_{2}-2\right) \equiv \bar{\Delta} .
$$

It follows from (3.10) that every state in a unitary representation of the superconformal group has $\bar{\Delta} \geq 0$. 
Following (3.3) we define

$$
\mathcal{I}^{W R}=\operatorname{Tr}_{R}\left[(-1)^{F} \exp (-\beta \bar{\Delta}+\bar{M})\right]
$$

where $\bar{M}$ is the part of the superconformal algebra that commutes with $\bar{Q}$ and $\bar{S}$.

The Cartan elements of this subalgebra are given in terms of those of the algebra by

$$
E^{\prime}=E+j_{2}, \quad j_{1}^{\prime}=j_{1}, \quad r^{\prime}=\frac{(m-1)\left(r+R_{m-1}\right)}{m}+\sum_{p=1}^{m-2} \frac{p}{m} R_{p}, \quad R_{k}^{\prime}=R_{k}
$$

Note that $r^{\prime}$ depends on $r$ and $R_{m-1}$ only through the combination $r+R_{m-1}$. We then find that the index (3.11) is zero on long representations and for $\mathbf{c}, \mathbf{b}$ representations it is equal to

$$
\begin{aligned}
\mathcal{I}^{W R}\left[\mathbf{b x}_{j_{1}, 0, r, R_{i}}\right] & =\chi_{s u b}[\overrightarrow{\vec{b}}] \\
\mathcal{I}^{W R}\left[\mathbf{c x}_{j_{1}, j_{2}, r, R_{i}}\right] & =(-1)^{2 j_{2}+1} \chi_{s u b}[\overrightarrow{\bar{c}}]
\end{aligned}
$$

where the representation of the subalgebra is specified by the vectors $\vec{b}, \vec{c}$ in the basis $\left[E^{\prime}, j_{1}^{\prime}, r^{\prime}, R_{k}^{\prime}\right]$ specified by (3.12).

$$
\begin{aligned}
& \overrightarrow{\vec{b}}=\left[-\frac{3}{2} r+2 r^{\prime}, j_{1}, r^{\prime}\left(r+R_{m-1}, R_{k}\right), R_{k}\right], \\
& \overrightarrow{\vec{c}}=\left[3+3\left(j_{2}-r / 2\right)+2 r^{\prime}, j_{1}, r^{\prime}\left(r+R_{m-1}, R_{k}\right), R_{k}\right] .
\end{aligned}
$$

where $r^{\prime}$ is the function in (3.12). We find that on a general representation (not necessarily irreducible) of the superconformal algebra, $\mathcal{I}^{W R}$ evaluates to

$$
\begin{aligned}
\mathcal{I}^{W R}[R]= & \sum_{j_{1}, r, R_{i}}\left(n\left[\mathbf{x b}_{j_{1}, 0, r, R_{k}, 0}\right] \chi_{s u b}\left[\overrightarrow{\bar{b}}_{0}\right]+n\left[\mathbf{x b}_{j_{1}, 0, r, R_{k}, 1}\right] \chi_{s u b}\left[\overrightarrow{\bar{b}}_{1}\right]\right) \\
& +\sum_{j_{1}, r^{\prime \prime}, R_{k}, N} I_{j_{1}, r^{\prime \prime}, R_{k}, N}^{R} \chi_{s u b}\left[\overrightarrow{\bar{c}}_{0}\right]
\end{aligned}
$$

Where $\overrightarrow{b_{0,1}}$ are given by (3.14) with $R_{m-1}=0,1$ respectively and $\overrightarrow{c_{0}}$ is given by (3.14) with $j_{2}=0, r=r^{\prime \prime}, R_{m-1}=N$. The quantities $n\left[\mathbf{x x}_{j_{1}, j_{2}, r, R_{i}}\right]$ in (3.9) are the number of copies of the irreducible representation, with listed quantum numbers, that appear in $R$, and $I_{j_{1}, r^{\prime \prime}, R_{k}, N}^{R}$ are the indices (2.19) made out of these numbers.

The main lesson we should extract from (3.9), (3.15) is that each of the indices defined in section two are multiplied by different $S U(1,2 \mid m-1)$ (or $S U(2,1 \mid m-1)$ ) characters in (3.9) (3.15). This shows that the Witten indices (3.3) (3.11) capture all the protected that follows from the supersymmetry algebra alone. 


\section{Computation of the Index in $\mathcal{N}=4$ Yang Mills on $S^{3}$}

\subsection{Weak Coupling}

We will now evaluate the index (3.3) for free $\mathcal{N}=4$ Yang Mills on $S^{3}$. In the Free theory this Index may be evaluated either by simply counting all gauge invariant states with $\Delta=0$ and specified values for other charges [2,3] or by evaluating a path integral [3]. The two methods give the same answer. We will give a very brief description of the path integral method, referring the reader to [3] for all details. One evaluates the path integral over the $\Delta=0$ modes of all the fields of the $\mathcal{N}=4$ theory on $S^{3} \times S^{1}$ with periodic boundary conditions for the fermions around $S^{1}$ (to deal with the $(-1)^{F}$ insertion) and twisted boundary conditions on all charged fields (to insert the appropriate chemical potentials). While the path integral over all other modes may be evaluated in the one loop approximation, the path integral over the zero mode of $A_{0}$ on this manifold must be dealt with exactly (as the integrand lacks a quadratic term for this mode, the integral over it is always strongly coupled at every nonzero coupling no matter how weak). Upon carefully setting up the problem one finds that the integral over $A_{0}$ is really an integral over the holonomy matrix $U$, and the index $\mathcal{I}^{W L}$ evaluates to

$$
\mathcal{I}_{Y M}=\int[d U] \exp \left\{\sum \frac{1}{m} f\left(t^{m}, y^{m}, u^{m}, w^{m}\right) \operatorname{tr}\left(U^{\dagger}\right)^{m} \operatorname{tr} U^{m}\right\}
$$

where $f(t, y, u, w)$ is the index $\mathcal{I}^{W L}$ evaluated on space of 'letters' or 'gluons' of the $\mathcal{N}=4$ Yang Mills theory. As a consequence, in order to complete our evaluation of the index (4.1) we must merely evaluate the single letter partition function $f$.

$f$ may be evaluated in many ways. Group theoretically, we note that the letters of Yang Mills theory transform in the 'fundamental' representation of the superconformal group (the representation whose quantum lowest weight state has quantum numbers $E=1$, $j_{1}=j_{2}=0, R_{1}=R_{3}=0$ and $\left.R_{2}=1\right) . f$ is simply the supertrace over this representation, which we have evaluated using group theoretic techniques in Appendix C.

It is useful, however, to re-derive this result in a more physical manner. The full set of single particle $\Delta=0$ operators in Yang Mills theory is given by the fields listed in Table 2 . below, acted on by an arbitrary numbers of the two derivatives $\partial_{+ \pm}$(see the last row of Table 2) modulo the single equation of motion listed in the second last row of Table 2. 
Table 2: Letters with $\Delta=0$

\begin{tabular}{l|l|l|l}
\hline Letter & $(-\mathbf{1})^{\mathbf{F}}\left[\mathbf{E} ; \mathbf{j}_{\mathbf{1}}, \mathbf{j}_{\mathbf{2}}\right]$ & {$\left[\mathbf{q}_{\mathbf{1}}, \mathbf{q}_{\mathbf{2}}, \mathbf{q}_{\mathbf{3}}\right]$} & {$\left[\mathbf{R}_{\mathbf{1}}, \mathbf{R}_{\mathbf{2}}, \mathbf{R}_{\mathbf{3}}\right]$} \\
\hline$X, Y, Z$ & {$[1,0,0]$} & {$[1,0,0]+$ cyclic } & {$[0,1,0]+[1,-1,1]+[1,0,-1]$} \\
\hline$\psi_{+, 0 ;-++}+$ cyc & $-\left[\frac{3}{2}, \frac{1}{2}, 0\right]$ & {$\left[-\frac{1}{2}, \frac{1}{2}, \frac{1}{2}\right]+$ cyc } & {$[1,-1,0],[0,1,-1],[0,0,1]$} \\
$\psi_{0, \pm,+++}$ & $-\left[\frac{3}{2}, 0, \pm \frac{1}{2}\right]$ & {$\left[\frac{1}{2}, \frac{1}{2}, \frac{1}{2}\right]$} & {$[1,0,0]$} \\
\hline$F_{++}$ & {$[2,1,0]$} & {$[0,0,0]$} & {$[0,0,0]$} \\
\hline$\partial_{++} \psi_{0,-;+++}+$ & {$\left[\frac{5}{2}, \frac{1}{2}, 0\right]$} & {$\left[\frac{1}{2}, \frac{1}{2}, \frac{1}{2}\right]$} & {$[1,0,0]$} \\
$\partial_{+-} \psi_{0,+;++}=$ & & & \\
0 & & {$[0,0,0]$} & {$[0,0,0]$} \\
\hline$\partial_{+ \pm}$ & {$\left[1, \frac{1}{2}, \pm \frac{1}{2}\right]$} & & \\
\hline \hline
\end{tabular}

In Table 2 we have listed both the $S U(4)$ Cartan charges $R_{1}, R_{2}, R_{3}$ used earlier in this paper, as well as the $S O(6)$ Cartan charges, $q_{1}, q_{2}, q_{3}$ (the eigenvalues in each of the 3 planes of the embedding $R^{6}$ ) of all fields.

To find $f$ we evaluate (3.3) by summing over the letters

$$
\begin{aligned}
f & =\sum_{\text {letters }}(-1)^{F} t^{2\left(E+j_{1}\right)} y^{2 j_{2}} v^{R_{2}} w^{R_{3}} \\
& =\frac{t^{2}\left(v+\frac{1}{w}+\frac{w}{v}\right)-t^{3}\left(y+\frac{1}{y}\right)-t^{4}\left(w+\frac{1}{v}+\frac{v}{w}\right)+2 t^{6}}{\left(1-t^{3} y\right)\left(1-\frac{t^{3}}{y}\right)} .
\end{aligned}
$$

Remarkably the expression for $1-f$ factorizes

$$
1-f=\frac{\left(1-t^{2} / w\right)\left(1-t^{2} w / v\right)\left(1-t^{2} v\right)}{\left(1-t^{3} y\right)\left(1-t^{3} / y\right)}
$$

The expression for $\mathcal{I}_{Y M}^{W L}$ is well defined (convergent) only if $t, y, v, w$ have values such that every contributing letter has a weight of modulus $<1$; applying this criterion to the three scalars and the two retained derivatives yields the restriction $t^{2} v<1, \quad t^{2} / w<1, \quad t^{2} v / w<$ 1, $t^{3} y<1, t^{3} / y>1$. It follows from th.3 that $f<1$ for all legal values of chemical potentials.

We will now proceed to evaluate the integral in (4.1), using saddle point techniques, in the large $N$ limit (note, however, that (4.1) is the exact formula valid for all $N$ ). To process this formula, we convert the integral over $U$ to an integral over its $N^{2}$ eigenvalues $e^{i \theta_{j}}$. We can conveniently summarize this information in a density distribution $\rho(\theta)$ with:

$$
\int_{-\pi}^{\pi} d \theta \rho(\theta)=1
$$


This generates an effective action for the eigenvalues given by [3]

$$
\begin{aligned}
S[\rho(\theta)] & =N^{2} \int d \theta_{1} \int d \theta_{2} \rho\left(\theta_{1}\right) \rho\left(\theta_{2}\right) V\left(\theta_{1}-\theta_{2}\right)= \\
& =\frac{N^{2}}{2 \pi} \sum_{n=1}^{\infty}\left|\rho_{n}\right|^{2} V_{n}(T),
\end{aligned}
$$

with

$$
\begin{aligned}
& V_{n}=\frac{2 \pi}{n}\left(1-f\left(t^{n}, y^{n}, u^{n}, w^{n}\right)\right), \\
& \rho_{n}=\int d \theta \rho(\theta) e^{i n \theta} .
\end{aligned}
$$

As $(1-f)$ is always positive for all allowed values of the chemical potential, it is clear that the action (4.5) is minimized by $\rho_{n}=0, n>0 ; \rho_{0}=1$. The classical value of the action vanishes on this saddle point, and the Index is given by the gaussian integral of the fluctuations of $\rho_{n}$ around zero. This allows us to write

$$
\left.\mathcal{I}_{Y M}^{W L}\right|_{N=\infty}=\prod_{n=1}^{\infty} \frac{1}{1-f\left(t^{n}, y^{n}, v^{n}, w^{n}\right)} .
$$

If we think about the 't Hooft limit of the theory it is also interesting to compute the index over single trace operators. This is given by

$$
\begin{aligned}
Z_{\text {s.t. }} & =-\sum_{r=1}^{\infty} \frac{\varphi(r)}{r} \log \left[1-f\left(t^{r}, y^{r}, v^{r}, w^{r}\right)\right] \\
& =\frac{t^{2} / w}{1-t^{2} / w}+\frac{v t^{2}}{1-v t^{2}}+\frac{t^{2} w / v}{1-t^{2} w / v}-\frac{t^{3} / y}{1-t^{3} / y}-\frac{t^{3} y}{1-t^{3} y}
\end{aligned}
$$

where $\varphi$ is the Euler Phi function and we used that $\sum_{r} \frac{\varphi(r)}{r} \log \left(1-x^{r}\right)=\frac{-x}{1-x}$. The result (4.7) is simply the multiparticle contribution that we get from (4.8).

Note that the action (4.5) vanished on its saddle point; as a consequence (4.7) is independent of $N$ in the large $N$ limit. This behavior, which is is reminiscent of the partition function of a large $N$ gauge theory in its confined phase, is true of (4.7) at all finite values of the chemical potential. In this respect the index $\mathcal{I}^{Y M}$ behaves in a qualitatively different manner from the free Yang Mills partition function over supersymmetric states (see the next section). This partition function displays confined behavior at large chemical potentials (analogous to low temperatures) but deconfined behavior (i.e. is of order $e^{N^{2}}$ ) at small chemical potentials (analogous to high temperature). It undergoes a sharp phase transition between these two behaviors at chemical potentials of order unity. Several recent 
studies of Yang Mills theory on compact manifolds have studied such phase transitions, and related them to the nucleation of black holes in bulk duals [2, 3, 26, 27, 28, 29, 30, 31]. The index $\mathcal{I}_{Y M}^{W L}$ does not undergo this phase transition, and is always in the 'confined' phase. We interpret this to mean that it never 'sees' the dual supersymmetric black hole phase.

At first sight we might think that this is a contradiction, since the black holes give a large entropy. On the other hand we are unaware of a clear argument which says that black holes should contribute to the index. For example, it is unclear whether the Euclidean black hole geometry should contribute to the path integral that computes the index. While the Lorentzian geometry of the black hole is completely smooth, if we compactify the Euclidean time direction with periodic boundary conditions for the spinors, then the corresponding circle shrinks to zero size at the horizon, which would represent a kind of singularity. See the next section and Appendix D for a mechanism for how this phenomenon (the excision of the black hole saddle point) might work in Lorentzian space.

We now present the expression for the Index in a new set of variables that are more symmetric, and for some purposes more convenient, in the study of Yang Mills theory. We will use these variables in the next section. Let us choose to parameterize charges in the subalgebra by

$$
J_{2}, L_{1}=E+q_{1}-q_{2}-q_{3}, L_{2}=E+q_{2}-q_{1}-q_{3}, L_{3}=E+q_{3}-q_{1}-q_{2} .
$$

Note that $L_{i}$ are positive for all Yang Mills letters. A simple change of basis, (see Appendix C) yields

$$
1-f=\frac{\left(1-e^{-2 \gamma_{1}}\right)\left(1-e^{-2 \gamma_{2}}\right)\left(1-e^{-2 \gamma_{3}}\right)}{\left(1-e^{-\zeta-\gamma_{1}-\gamma_{2}-\gamma_{3}}\right)\left(1-e^{+\zeta-\gamma_{1}-\gamma_{2}-\gamma_{3}}\right)}
$$

where

$$
f=\sum_{\text {letters }}(-1)^{F} e^{\gamma_{1} L_{1}+\gamma_{2} L_{2}+\gamma_{3} L_{3}+2 \zeta j_{2}}
$$

In section six we will write an explicit exact formulas for the index (4.1) for $\gamma_{3}=\infty$. Further studies on the spectrum of free Yang Mills can be found in [32, 33, 34, 35].

\subsection{Strong Coupling}

According to the AdS/CFT correspondence, $\mathcal{N}=4$ Yang Mills theory on $S^{3}$ at large $N$ and large $\lambda$ has a dual description as a weakly coupled IIB theory on the large radius $A d S_{5} \times S^{5}$. At fixed energies in the large $N$ limit, the spectrum of the bulk dual is a gas of free gravitons, plus superpartners, on $A d S_{5} \times S^{5}$. In this subsection section we will 
compute the index $\mathcal{I}_{Y M}^{W L}$ over this gas of masssless particles, and find perfect agreement with (4.7).

Note that states with energies of order one do not always dominate the partition function at chemical potentials of unit order. At small values of the chemical potential, the usual partition function of strongly coupled Yang Mills theory is dominated by black holes. However, as we have explained in the previous subsection, we do not see an argument for the black hole saddle point to contribute to the Index, and apparently it does not.

We now turn to the computation. When the spectrum of (single particle) supergravitons of Type $I I B$ supergravity compactified on $A d S_{5} \times S^{5}$ is organized into representations of the superconformal group, we obtain representations that are built on a lowest weight state that is a $S U(2) \times S U(2)$ in the $(n, 0,0)_{S O(6)}=(0, n, 0)_{S U(4)}$ representation of the R-symmetry group [36]. The representation with $n=1$ is the Yang-Mills multiplet. The representation with $n=2$ is called the 'supergraviton' representation. These representations preserve 8 of 16 supersymmetries. In the language of section 2 , they are of the form bb. When restricted to $\Delta=0$, they yield a representation of the subalgebra that we shall call $S_{n}$. $S_{n}$ has lowest weights $E^{\prime}=n, j_{2}=0, R_{2}=n, R_{3}=0$. The states of $S_{n}$ are tabulated explicitly in appendix C. The state content of $n=1$ is somewhat different and is tabulated separately. This can also be found by looking at the list of Kaluza Klein modes in 36 .

The index on single-particle states may now be calculated in a straightforward manner. The supercharacter of $S_{n}$ may be read off from the appendix and is given by

$$
\chi_{S n}=\frac{\left(t^{2 n} \chi_{n, 0}^{S U(3)}(v, w)-t^{2 n+1} \chi_{n-1,0}^{S U(3)}(v, w)(y+1 / y)+\ldots\right)}{\left(1-t^{3} y\right)\left(1-t^{3} / y\right)} .
$$

The $S U(3)$ character that occurs above is described by the Weyl Character Formula described in the Appendix C. To obtain the index, we simply need to calculate

$$
\mathcal{I}_{s p}=\sum_{n=2}^{\infty} \chi_{S n}+\chi_{S 1}
$$

The sums in (4.13) are all geometric and are easily performed, yielding the single particle contribution

$$
\mathcal{I}_{s p}=\frac{t^{2} / w}{1-t^{2} / w}+\frac{v t^{2}}{1-v t^{2}}+\frac{t^{2} w / v}{1-t^{2} w / v}-\frac{t^{3} / y}{1-t^{3} / y}-\frac{t^{3} y}{1-t^{3} y}
$$

This matches precisely (4.8). 
To obtain the index for the Fock-space of gravitons we use the formula, justified in the Appendix C, that relates the index of one particle to the index of the Fock Space.

$$
\begin{aligned}
\mathcal{I}_{\text {grav }}^{W L} & =\exp \left[\sum_{n} \frac{1}{n} \mathcal{I}_{\text {sp }}\left[t^{n}, v^{n}, w^{n}, y^{n}\right]\right] \\
& =\prod_{n=1}^{\infty} \frac{\left(1-t^{3 n} / y^{n}\right)\left(1-t^{3 n} y^{n}\right)}{\left(1-t^{2 n} / w^{n}\right)\left(1-v^{n} t^{2 n}\right)\left(1-t^{2 n} w^{n} / v^{n}\right)}
\end{aligned}
$$

in perfect agreement with (4.7).

Finally, let us point out that the value of the index is the same in $\mathcal{N}=1$ marginal deformations of $\mathcal{N}=4.11$

\section{The partition function over BPS states}

In this section we will compute the partition function over BPS states that are annihilated by $Q$ and $S$ in $\mathcal{N}=4$ Yang Mills at zero coupling and strong coupling. We perform the first computation using the free Yang Mills action, and the second computation using gravity and the AdS/CFT correspondence, together with a certain plausible assumption. Specifically, we assume that the supersymmetric density of states at large charges is dominated by the supersymmetric black holes of [6, 7, 8,

At small values of chemical potentials (when these supersymmetric partition functions are dominated by charges that are large in units of $N^{2}$ ) we find that these partition functions are qualitatively similar at weak and strong coupling but differ in detail, in these two limits. Moreover, each of these partition functions differs qualitatively from index computed in the previous section.

Before turning to the computation, it may be useful to give a more formal description of the BPS states annihilated by $Q$ and $S . Q$ may formally be thought of as an exterior derivative $d$, its Hermitian conjugate $S$ is then $d^{*}$ and $\Delta$ is the Laplacian $d d^{*}+d^{*} d$. States with $\Delta=0$ are harmonic forms that, according to standard arguments (see [37], those arguments may all be reworded in the language of $Q$ and $S$ and Hilbert spaces) are in one to one correspondence with the cohomology of $Q . \mathcal{I}^{W L}$, the $(-1)^{\text {degree }}$ weighted partition function over this cohomology is simply the (weighted) Euler Character over this cohomology.

11 These theories have the superpotential $\operatorname{Tr}\left[e^{\beta} \phi_{1} \phi_{2} \phi_{3}-e^{-\beta} \phi_{1} \phi_{3} \phi_{2}+c\left(\phi_{1}^{3}+\phi_{2}^{3}+\phi_{3}^{3}\right)\right]$. If $c$ is nonzero, then we should set all chemical potentials $\gamma_{i}$ to be equal in the original $\mathcal{N}=4$ result, since we loose two of the $U(1)$ symmetries. 


\subsection{Partition Function at $\Delta=0$ in Free Yang Mills}

Let

$$
Z_{\text {free }}=\operatorname{Tr}_{\Delta=0}\left[x^{2 H} e^{\mu_{1} q_{1}+\mu_{2} q_{2}+\mu_{3} q_{3}+2 \zeta J_{2}}\right]
$$

where $x=e^{\frac{-\beta}{2}}$, and $q_{1}, q_{2}, q_{3}$ correspond to the $S O(6)$ Cartan charges (related to $R_{1}, R_{2}, R_{3}$ by the formulas in Appendix C). In Free Yang Mills theory this partition function is easily computed along the lines described in subsection 4.1; the final answer is given by the formula [2,3]

$$
Z=\int D U \exp \left[\sum_{n}\left(f_{B}\left(x^{n}, n \mu_{i}, n \zeta\right)+(-1)^{n+1} f_{F}\left(x^{n}, n \mu_{i}, n \zeta\right)\right) \frac{\operatorname{Tr} U^{n} \operatorname{Tr} U^{-n}}{n}\right]
$$

where $U$ is a unitary matrix and the relevant 'letter partition functions' are given by

$$
\begin{aligned}
& f_{B}=\frac{\left(e^{\mu_{1}}+e^{\mu_{2}}+e^{\mu_{3}}\right) x^{2}+x^{4}}{\left(1-x^{2} e^{\zeta}\right)\left(1-x^{2} e^{-\zeta}\right)} \\
& f_{F} \frac{x^{3}\left(2 \cosh \zeta e^{\frac{\mu_{1}+\mu_{2}+\mu_{3}}{2}}+e^{\frac{\mu_{1}+\mu_{2}-\mu_{3}}{2}}+e^{\frac{\mu_{1}-\mu_{2}+\mu_{3}}{2}}+e^{\frac{-\mu_{1}+\mu_{2}+\mu_{3}}{2}}\right)-x^{5} e^{\frac{\mu_{1}+\mu_{2}+\mu_{3}}{2}}}{\left(1-x^{2} e^{\zeta}\right)\left(1-x^{2} e^{-\zeta}\right)} .
\end{aligned}
$$

As explained in the previous section, (5.2) and (5.3) describe a partition function that undergoes a phase transition at finite values of chemical potentials. For chemical potentials such that $f_{B}+f_{F}<1$, the integral in (5.2) is dominated by a saddle point on which $\left|\operatorname{Tr} U^{n}\right|=0$ for all $n$. In this phase the partition function is obtained from the one loop integral about the saddle point (as in section 4.1) and is independent of $N$ in the large $N$ limit. The density of states in this phase grows exponentially with energy, $\rho(E) \propto e^{\beta_{H} E}$ where $\beta_{H}=-\ln \left(\frac{7-3 \sqrt{5}}{2}\right)=1.925$ and the system undergoes a phase transition when the effective inverse temperature becomes smaller than $\beta_{H}$ (e.g., on setting all other chemical potentials to zero, this happens at $x=e^{\frac{-\beta_{H}}{2}}$ ).

At smaller values of chemical potentials (5.2) is dominated by a new saddle point. In particular, in the limit $\zeta \ll 1$ and $\beta \ll 1$, the integral over $U$ in (5.1) is dominated by a saddle point on which $\operatorname{Tr} U^{n} \operatorname{Tr} U^{-n}=N^{2}$ for all $n$, the partition function reduces to

$$
\ln Z=N^{2} \sum_{n} \frac{1}{n}\left[f_{B}\left(x^{n}, n \mu_{i}, n \zeta\right)+(-1)^{n+1} f_{F}\left(x^{n}, n \mu_{i}, n \zeta\right)\right] .
$$


In the rest of this subsection we will, for simplicity, set $\mu_{1}=\mu_{2}=\mu_{3}=\mu$ and thereby focus on that part of cohomology with $q_{1}=q_{2}=q_{3} \equiv q$. The relevant letter partition functions reduce to

$$
\begin{aligned}
f_{B} & =\frac{3 e^{\mu} x^{2}+x^{4}}{\left(1-x^{2} e^{\zeta}\right)\left(1-x^{2} e^{-\zeta}\right)} \\
f_{F} & =\frac{\left(e^{\frac{3 \mu}{2}}\left(2 \cosh \zeta-x^{2}\right)+3 e^{\frac{\mu}{2}}\right) x^{3}}{\left(1-x^{2} e^{\zeta}\right)\left(1-x^{2} e^{-\zeta}\right)}
\end{aligned}
$$

In the limit $\beta \ll 1, \zeta \ll 1($ 5.4 $)$ reduces to

$$
\ln Z=N^{2} \frac{1}{\left(\beta^{2}-\zeta^{2}\right)} f(\mu)
$$

where

$$
f(\mu)=\left(\zeta(3)+3 P l\left(3, e^{\mu}\right)-3 P l\left(3,-e^{\frac{\mu}{2}}\right)-P l\left(3,-e^{\frac{3 \mu}{2}}\right)\right)
$$

and the PolyLog function is defined by

$$
P l(m, x)=\sum_{n=1}^{\infty} \frac{x^{n}}{n^{m}}
$$

This partition function describes a system with energy $E$, angular momentum $j_{2}, S O(6)$ charge $(q, q, q)$ and entropy $S$ given by 12

$$
\begin{aligned}
\frac{2 j_{1}}{N^{2}} \sim \frac{E}{N^{2}} & =2 \frac{\beta f(\mu)}{\left(\beta^{2}-\zeta^{2}\right)^{2}}, \\
\frac{2 j_{2}}{N^{2}} & =2 \frac{\zeta f(\mu)}{\left(\beta^{2}-\zeta^{2}\right)^{2}} \\
\frac{q}{N^{2}} & =\frac{g(\mu)}{\beta^{2}-\zeta^{2}} \\
\frac{S}{N^{2}} & =\frac{3 f(\mu)-\mu g(\mu)}{\beta^{2}-\zeta^{2}}
\end{aligned}
$$

where

$$
g(\mu)=\frac{f^{\prime}(\mu)}{3}=\left(P l\left(2, e^{\mu}\right)-\frac{1}{2} P l\left(2,-e^{\frac{\mu}{2}}\right)-\frac{1}{2} P l\left(2,-e^{\frac{3 \mu}{2}}\right)\right) .
$$

12 Physically, the equations below describe Free Yang Mills theory at fixed values of charges in the limit $T \rightarrow 0$ ( $T$ is the temperature). In the free theory this limit retains only supersymmetric states at all values of charges. On the other hand the black holes in [6, 7, 8] are supersymmetric in the same limit only for a subfamily of charges. See the next section for more discussion on this puzzling difference. 
We see that for high temperatures, this partition function looks like a gas of massless particles in $2+1$ dimensions. Note that in this limit $E \sim 2 j_{1} \gg q$.

We will sometimes be interested in the partition function with only those chemical potentials turned on that couple to charges that commute with $Q$ and $S$. This is achieved if we choose $\mu=\frac{\beta}{3}$. In the limit $\beta \ll 1, \zeta \ll 1$ we have $\mu \ll 1$ and the partition function and charges are given by (5.6) and (5.9) with $\mu \sim 0$; note that $f(0)=7 \zeta(3)$ and $g(0)=\frac{\pi^{2}}{4}$.

Note that, although the index $\mathcal{I}_{Y M}^{W L}$ and the cohomological partition function $Z_{\text {free }}$ both traces over $Q$ cohomology, the final results for these two quantities in Free Yang Mills theory are rather different. For instance, at finite but small values of chemical potentials, $\ln Z_{\text {free }}$ is proportional to $N^{2}$ (see (5.6)) while $\mathcal{I}_{Y M}^{W L}$ is independent of $N$ (see (4.7)). Clearly cancellations stemming from the fluctuating sign in the definition of $\mathcal{I}_{Y M}^{W L}$ cause the index to see a smaller effective number of states. In Appendix D we explain, in more detail, how this might come about.

\subsection{Cohomology at Strong Coupling: Low energies}

We now turn to the study of $Q$ cohomology at strong coupling and low energies. In this limit the cohomology is simply that of the free gas of supergravitons in $A d S_{5} \times S^{5}$, and may be evaluated following the method of subsection subsection 4.2 . We will calculate the quantity

$$
Z=\operatorname{Tr}\left[x^{2 H} z^{2 J_{1}} y^{2 J_{2}} v^{R_{2}} w^{R_{3}}\right]
$$

over the supergraviton representations restricted to states of $\Delta=0$. We recall that the single particle states form an infinite series of short reps of the $N=4$ superconformal algebra where the primary is a lorentz scalar with energy $n$ with R-charges $[0, n, 0]$. 
The trace over single particle states may be easily calculated. The answer is 13

$$
\begin{aligned}
Z_{\mathrm{sp}} & =\frac{\text { num }_{\text {bos }}+\operatorname{num}_{\text {fer }}}{\operatorname{den}} \\
\operatorname{den} & =\left(1-x^{2} / w\right)\left(1-x^{2} v\right)\left(1-x^{2} w / v\right)\left(1-x^{2} z / y\right)\left(1-x^{2} z y\right) \\
\operatorname{num}_{\text {fer }} & =x^{3} / y+x^{3} y+x^{3} z / v+v x^{3} z / w+w x^{3} z-2 x^{5} z \\
& +v x^{7} z+x^{7} z / w+w x^{7} z / v+x^{7} z^{2} / y+x^{7} z^{2} y \\
\text { num }_{\text {bos }} & =v x^{2}+x^{2} / w+w x^{2} / v-x^{4} / v-v x^{4} / w-w x^{4} \\
& +2 x^{6}+x^{6} z /(y v)+v x^{6} z /(w y)+w x^{6} z / y-x^{8} z / y \\
& +x^{6} z y / v+v x^{6} z y / w+w x^{6} z y-x^{8} z y+x^{4} z^{2}+x^{10} z^{2}
\end{aligned}
$$

The full (multi particle) partition function over supersymmetric states may be obtained by applying the formulas of Bose and Fermi statistics to (5.13).

Special limits of (5.13) will be of interest in the next section. For instance, the limit $z \rightarrow 0$ focus on states with $\Delta=0$ and $j_{1}=0$, i.e. (1/8) BPS states. In this limit (5.13) becomes

$$
\begin{aligned}
Z_{\mathrm{bos}-\mathrm{sp}}^{1 / 8} & =\frac{1-\left(1-x^{2} / w\right)\left(1-v x^{2}\right)\left(1-w x^{2} / v\right)+x^{6}}{\left(1-x^{2} / w\right)\left(1-v x^{2}\right)\left(1-w x^{2}\right)} \\
Z_{\mathrm{fer}-\mathrm{sp}}^{1 / 8} & =\frac{x^{3}(y+1 / y)}{\left(1-x^{2} / w\right)\left(1-v x^{2}\right)\left(1-w x^{2} / v\right)}
\end{aligned}
$$

In terms of the $\gamma_{i}$ variables introduced at the end of subsection 4.1

$$
\begin{aligned}
Z_{\text {bos-sp }}^{1 / 8} & =\frac{1-\left(1-e^{-2 \gamma_{1}}\right)\left(1-e^{-2 \gamma_{2}}\right)\left(1-e^{-2 \gamma_{3}}\right)+e^{-2\left(\gamma_{1}+\gamma_{2}+\gamma_{3}\right)}}{\left(1-e^{-2 \gamma_{1}}\right)\left(1-e^{-2 \gamma_{2}}\right)\left(1-e^{-2 \gamma_{3}}\right)} \\
Z_{\text {fer-sp }}^{1 / 8} & =\frac{e^{-\gamma_{1}-\gamma_{2}-\gamma_{3}}\left[e^{\zeta}+e^{-\zeta}\right]}{\left(1-e^{-2 \gamma_{1}}\right)\left(1-e^{-2 \gamma_{2}}\right)\left(1-e^{-2 \gamma_{3}}\right)}
\end{aligned}
$$

13 In the notation of the previous subsection, with $y=e^{\zeta}$,

$$
\begin{aligned}
Z_{s p}^{\mathrm{res}} & =\operatorname{Tr}\left[x^{2 H} y^{2 J_{2}} u^{2} \sum q_{i}\right]=\frac{\text { num }_{\mathrm{bos}}^{\mathrm{res}}+\text { num }_{\mathrm{fer}}^{\mathrm{res}}}{\operatorname{den}^{\mathrm{res}}} \\
\operatorname{den} & =\left(1-x^{2} u^{2}\right)^{3}\left(1-x^{2} / y\right)\left(1-x^{2} y\right) \\
\text { num }_{\mathrm{fer}} & =3 u x^{3}-2 u^{3} x^{5}+3 u^{5} x^{7}+\left(u^{3} x^{3}\right) / y+\left(u^{3} x^{7}\right) / y \\
& +u^{3} x^{3} y+u^{3} x^{7} y \\
\text { num }_{\mathrm{bos}} & =3 u^{2} x^{2}+x^{4}-3 u^{4} x^{4}+2 u^{6} x^{6}+u^{6} x^{10} \\
& +\left(3 u^{4} x^{6}\right) / y-\left(u^{6} x^{8}\right) / y+3 u^{4} x^{6} y-u^{6} x^{8} y
\end{aligned}
$$


Applying the formulas for Bose and Fermi statistics, it is now easy to see that the partition function over the Fock space, including multi-particle states, is given by

$$
Z^{1 / 8}\left(\zeta, \gamma_{1}, \gamma_{2}, \gamma_{3}\right)=\prod_{n, m, r=0}^{\infty} \frac{\prod_{s= \pm 1}\left(1+e^{s \zeta} e^{-(2 n+1) \gamma_{1}-(2 m+1) \gamma_{2}-(2 r+1) \gamma_{3}}\right)}{\left(1-e^{-2 n \gamma_{1}-2 m \gamma_{2}-2 r \gamma_{3}}\right)\left(1-e^{\left.-(2 n+2) \gamma_{1}-(2 m+2) \gamma_{2}-(2 m+2) \gamma_{3}\right)}\right.}
$$

Finally, in order to compute the rate of growth of the cohomological density of states with respect to energy, we set $z, y, v, w \rightarrow 1$. This gives the "blind" single particle partition function which is

$$
\begin{aligned}
Z_{\mathrm{bos}-\mathrm{sp}}^{\mathrm{bl}} & =\frac{x^{2}\left(3-2 x^{2}+8 x^{4}-2 x^{6}+x^{8}\right)}{\left(1-x^{2}\right)^{5}} \\
Z_{\mathrm{fer}-\mathrm{sp}}^{\mathrm{bl}} & =\frac{x^{3}\left(5-2 x^{2}+5 x^{4}\right)}{\left(1-x^{2}\right)^{5}}
\end{aligned}
$$

The full partition function is given by

$$
Z^{\mathrm{bl}}=\exp \left[\sum_{n} \frac{Z_{\mathrm{bos}-\mathrm{sp}}^{\mathrm{bl}}\left(x^{n}\right)+(-1)^{n+1} Z_{\mathrm{fer}-\mathrm{sp}}^{\mathrm{bl}}\left(x^{n}\right)}{n}\right]
$$

Let

$$
x=e^{\frac{-\beta}{2}} .
$$

At small $\beta$ this partition function is approximately given by

$$
\ln Z=\frac{63 \zeta(6)}{4 \beta^{5}}
$$

It follows that the entropy as a function of energy is given by

$$
S(E)=h \log n(E) \sim \frac{6}{5}\left(\frac{315 \zeta(6)}{4}\right)^{\frac{1}{6}} E^{5 / 6} \approx 2.49 E^{5 / 6}
$$

Note that this is slower than the exponential growth of the same quantity at zero coupling.

\subsection{Cohomology at Strong Coupling: High Energies}

Gutowski and Reall [6,7], and Chong, Cvetic, Lu and Pope [8] have found a set of supersymmetric black holes in global $A d S_{5} \times S^{5}$, that are annihilated by the supercharges $Q$ and $S$. These black holes presumably dominate the supersymmetric cohomology at energies of order $N^{2}$ or larger. In this subsection we will translate the thermodynamics of these supersymmetric black holes to gauge theory language, and compare the results with the free cohomology of subsection 5.1. 
Restricting to black holes with $q_{1}=q_{2}=q_{3}=q$ these solutions constitute a two parameter set of solutions, with thermodynamic charges, translated to Yang Mills Language via the AdS/CFT dictionary 14 ,

$$
\begin{aligned}
\frac{E}{N^{2}} & =(a+b) \frac{[(1-a)(1-b)+(1+a)(1+b)(2-a-b)]}{2(1-a)^{2}(1-b)^{2}} \\
\frac{j_{1}+j_{2}}{N^{2}} & =\frac{(a+b)(2 a+b+a b)}{2(1-a)^{2}(1-b)} \\
\frac{j_{1}-j_{2}}{N^{2}} & =\frac{(a+b)(a+2 b+a b)}{2(1-a)(1-b)^{2}} \\
\frac{q}{N^{2}} & =\frac{(a+b)}{2(1-a)(1-b)} \\
\frac{S}{N^{2}} & =\frac{\pi(a+b) \sqrt{a+b+a b}}{(1-a)(1-b)} .
\end{aligned}
$$

Setting $a=1-\left(\beta^{\prime}+\zeta^{\prime}\right)$ and $b=1-\left(\beta^{\prime}-\zeta^{\prime}\right)$, and assuming $\beta^{\prime} \ll 1, \zeta^{\prime} \ll 1$, (5.22) reduces to

$$
\begin{aligned}
\frac{2 j_{1}}{N^{2}} \sim \frac{E}{N^{2}} & \sim \frac{8 \beta^{\prime}}{\left(\beta^{\prime 2}-\zeta^{\prime 2}\right)^{2}} \\
\frac{2 j_{2}}{N^{2}} & \sim \frac{-8 \zeta^{\prime}}{\left(\beta^{\prime 2}-\zeta^{\prime 2}\right)^{2}} \\
\frac{q}{N^{2}} & \sim \frac{1}{\beta^{\prime 2}-\zeta^{\prime 2}} \\
\frac{S}{N^{2}} & \sim \frac{2 \sqrt{3} \pi}{\beta^{\prime 2}-\zeta^{\prime 2}}
\end{aligned}
$$

Equations (5.22) and (5.9) are have some clear similarities 15 in form, but also have one important qualitative difference. (5.9) has one additional parameter absent in (5.22). After setting the three $S O(6)$ charges equal the $Q$ cohomology is parametrized by 3 charges, whereas only a two parameter set of supersymmetric black hole solutions are available.

We should emphasize that in the generic, non BPS, situation black hole solutions are available for all values of the 4 parameters $q, j_{2}, j_{1}$ and $E$ [8]. It is thus possible to continuously lower the black hole energy while keeping $q, j_{2}$ and $j_{1}$ fixed at arbitrary values.

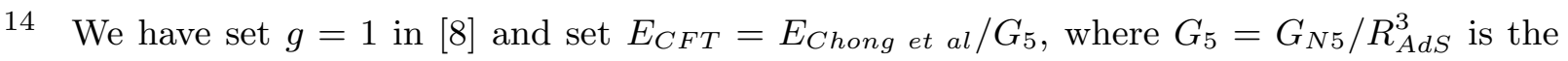
value of Newton's constant in units where the $A d S_{5}$ radius is set to one. $S_{\text {here }}=S_{\text {Chong et al }} / G_{5}$. For $\mathcal{N}=4$ Yang Mills we have $G_{5}=\frac{\pi}{2 N^{2}}$. To convert formulas in [6.7] simply set this value for the five dimensional Newton constant in their expressions.

15 This observation has also been made by H. Reall and R. Roiban. 
The temperature of the black hole decreases as we lower its energy, until it eventually goes to zero at a minimum energy. However the extremal black hole thus obtained is supersymmetric (its mass saturates the supersymmetric bound) only on a 2 dimensional surface in the 3 dimensional space of charges parameterized by $q, j_{2}$ and $j_{1}$. For every other combination of charges the zero temperature black holes are not supersymmetric (their mass is larger than the BPS bound). We are unsure how this should be interpreted 16 . It is possible that, for other combinations of charges, the cohomology is captured by as yet undiscovered supersymmetric black solutions.

In order to compare the cohomologies in (5.9) and (5.22) in more detail, we choose $\mu$ in (5.9) so that the equations for $E / N^{2}$ and $q / N^{2}$ in (5.9) and (5.22) become identical (after a rescaling of $\beta^{\prime}$ and $\zeta^{\prime}$ ). This is achieved provided that

$$
f\left(\mu_{c}\right)^{2}=16 g\left(\mu_{c}\right)^{3}
$$

This equation is easy to solve numerically. We find $\mu_{c}=-0.50366 \pm .00001$ and that $f\left(\mu_{c}\right)=5.7765, g\left(\mu_{c}\right)=1.2776$. Plugging in $\mu=\mu_{c}$ into the entropy formula in (5.9) we then find

$$
\frac{S_{\text {Field }}}{S_{\text {Black-Hole }}}=\frac{3 \frac{f(\mu)}{g(\mu)}-\mu}{2 \sqrt{3} \pi}=1.2927
$$

Another way to compare (5.9) and (5.22) is the following. First notice that the charge $q$ is much smaller than the energy in this limit, $q \ll E$. Let us set $\mu=\beta / 3$ which is the value that we have in the index (though we do not insert the $(-1)^{F}$ we have in the index). Since we are taking the limit where $\beta$ is small we can evaluate $f$ in (5.9) at zero, $f(0)=7 \zeta(3)$. By comparing the energies and entropies in (5.9) and (5.22) and writing the free energy as $E=N^{2} c \beta^{-3}$, where $c$ is a "central charge" that measures the number of degrees of freedom. Then we can compute

$$
\frac{c_{\text {gravity }}}{c_{\text {free-field-theory }}}=\frac{\pi^{3}}{14 \zeta(3) 3^{3 / 2}} \sim 0.35458 \ldots
$$

It is comforting that this value is lower than one since we expect that interactions would remove BPS states rather than adding new ones. A similar qualitative agreement between the weak and strong coupling was observed between the high temperature limit of uncharged black holes and the free Yang Mills theory [38], where the ratio (5.26) is 3/4. Note that for $\mu=\beta / 3$ we can approximate $g$ in the expression for the charge in (5.9) by $g(0) \neq 0$. This agrees qualitatively with the expression coming from black holes.

16 Note that our Index $\mathcal{I}_{Y M}^{W L}$, when specialized to states with $q_{1}=q_{2}=q_{3}$, also depends on two rather than 3 parameters. 


\subsection{Cohomology at Intermediate Energies: Giant Gravitons and Small Black Holes}

Let us set $j_{2}=0$ or $a=b$ in (5.22). We then expand the resulting expression for low values of $a$.

$$
\begin{aligned}
\frac{E}{N^{2}} & \sim 3 a \sim 3 \frac{q}{N^{2}} \\
\frac{j_{1}}{N^{2}} & \sim 3 a^{2} \\
\frac{S}{N^{2}} & \sim 2 \pi \sqrt{2} a^{3 / 2} .
\end{aligned}
$$

It is possible to count the entropy of these black holes using D-branes in $A d S$. This is not the same problem as counting them in the field theory, but perhaps these results might be a good hint for the kind of states that we should look at in the field theory.

In the small charge limit the black holes looks very similar to the black holes that appear in toroidal compactifications of type IIB on $T^{5}$. Let us recall first how the entropy of these black holes is counted [9]. We view the black holes as arising from two sets of intersecting D3 branes $n_{3}$ and $n_{3}^{\prime}$ which intersect along a circle which is one of the circles of $T^{5}$. One can then add momentum $L_{0}$ along this circle. Their entropy is given by $S=2 \pi \sqrt{n_{3} n_{3}^{\prime} L_{0}}$. This entropy arises as follows. Let us focus on the $T^{4}$ that is orthogonal to the common circle. The D3 branes can form any homomorphic surface on this $T^{4}$. The number of complex moduli of these surfaces goes as $n_{3} n_{3}^{\prime}$. There is an equal number of Wilson lines and there are $4 n_{3} n_{3}^{\prime}$ fermions. This gives central charge $c=6 n_{3} n_{3}^{\prime}$ and then using the Cardy formula we get the entropy.

We will now repeat the same counting for small black holes in our context. First we recall that the theory contains giant graviton D3 branes which can carry some of the charge. Let us recall the description in [39] for giant gravitons on the 5-sphere. We take an arbitrary holomorphic 2-complex dimensional surface in $C^{3}$ and we intersect it with $\sum\left|z_{i}\right|=1$. This gives a 3 -real dimensional surface on $S^{5}$ which will be a giant graviton. Let us focus first on surfaces that are invariant under $\psi$ translations, where $\psi$ is an angle that rotates all $z_{i} \rightarrow e^{i \psi} z_{i}$. The holomorphic surface in $C^{3}$ is specified by a homogeneous polynomial of degree $n$.

$$
\sum_{n_{1}+n_{2}+n_{3}=n} C_{n_{1}, n_{2}, n_{3}} z_{1}^{n_{1}} z_{2}^{n_{2}} z_{3}^{n_{3}}=0
$$

Think of $S^{5}$ as an $S^{1}$ fibration on $C P^{2}$. Then (5.28) defines a holomorphic surface in $C P^{2}$ and the resulting giant graviton on $S^{5}$ consists of this surface plus the $S^{1}$ fiber which is 
parametrized by the angle $\psi$. For example, the maximum size giant graviton that wraps an $S^{3}$ in $S^{5}$ [40] corresponds to the equation $z_{1}=0$. The number of complex parameters characterizing the curve (5.28) goes as

$$
d \sim n^{2} / 2
$$

In order to compute the charge under the $U(1)$ gauge field that performs translations in $\psi$ we need know to how many times this curve wraps the $C P^{1}$ inside $C P^{2}$ [41, 42]. It is easy to see that this number is $n$. The amount of wrapping of this curve over the $C P^{1}$ in $C P^{2}$ is $n$. So the total charge under the generator $J$ that rotates all the angles is

$$
\widehat{q}=\sum_{i} q_{i}=N n
$$

We define overall $U(1)$ charge $\widehat{q}$ to be the change in phase when we shift $\psi \rightarrow \psi+2 \pi$. So we have that $e^{\hat{i q} \psi}$ is the shift in phase for a state of charge $\widehat{q}$.

Our strategy is as follows. The total charge that we have at our disposal is $\widehat{q}=3 q$. We split it as $\widehat{q}=(3 q-n N)+n N$. The second term will be realized by $n$ giant gravitons and the first by momentum along $\psi$. In other words, the $n$ giant gravitons are D3 branes that are intersecting at points on the $C P^{2}$ (and form a smooth surface (5.28)) and are coincident along the fiber parametrized by $\psi$. We have many moduli of this configurations counted by (5.29). The momentum $L_{0} \equiv(3 q-n N)$ will be carried by these oscillations. In other words the D3 branes wrapped along $\psi$ give us an effective string with central charge $c=6 d=3 n^{2}$.

Then the entropy is

$$
S=2 \pi \sqrt{c L_{0} / 6}=2 \pi \sqrt{\frac{1}{2} n^{2}(3 q-n N)}
$$

Note that we have not said anything about the value of $n$. We now maximize the entropy with respect to $n$ we get

$$
n=2 q / N
$$

and substituting in (5.31) we obtain

$$
S=2 \pi \sqrt{2} \frac{q^{3 / 2}}{N}
$$

in agreement with (5.27). 
Notice, however that there is an important difference between the computation that is done here and the usual computation for the $D 1 D 5 p$ system. In the latter case it is possible to vary the parameters of the compactification to go to a regime where the amount of energy contained in momentum is much smaller than the energy of the Dbranes, which is a necessary condition for being able to view the momentum as producing small oscillations on the D-branes. In the discussion of this section it is not possible to satisfy this condition. Equation (5.32) implies that the energy contained in oscillations of the branes is comparable to the brane tensions, and there is no obvious parameter that we can adjust to change this fact 17 . As a consequence the discussion of this section falls short of qualifying as a completely satisfactory derivation of (5.33) (note, nonetheless, that all factors work bang on).

This point of view lets us also heuristically understand why we need to have angular momentum $j_{1}$. This arises as follows. The system we had above is very similar to the $D 1 D 5 p$ system in the NS sector, since the fermions are anti-periodic in the $\psi$ direction. Recall that the $D 1 D 5 p$ black hole has $j_{1}=0$ [43] (though $j_{2}$ can be non-zero 18 ) and this black hole naturally arises in the Ramond sector. When we perform a spectral flow to the NS sector we get $j_{1}=\frac{c}{12}$. In our case, we cannot choose the fermions to be periodic along $\psi$ due to the way the circle is fibered over $C P^{2}$. However, writing down the same formula as we had for the $D 1 D 5 p$ in the NS sector we would get $j_{1}=\frac{c}{12}=\frac{n^{2}}{4}=\frac{q^{2}}{N^{2}}$. On the other hand we get $j_{1}=3 \frac{q^{2}}{N^{2}}$ from (5.27), which has a different numerical coefficient. It would be nice to compute $j_{1}$ properly and see whether it agrees with the black hole answer.

\section{Partition Functions over $\frac{1}{2}, \frac{1}{4}^{t h}$ and $\frac{1}{8}^{t h}$ BPS States}

In this section we will study the partition function over $\frac{1}{8}^{\text {th }}$, a quarter and half BPS supersymmetric states in $\mathcal{N}=4$ Yang Mills. We will compute these partition functions in free Yang Mills, at weak coupling using naive classical formulas, and at strong coupling using the AdS/CFT correspondence. In the case of quarter and $\frac{1}{8}^{\text {th }}$ BPS states, our free and weak coupling partition functions are discontinuously different. However the weak

17 One would like to increase the radius of the $\psi$ circle without changing anything else, but this would not be a solution to the gravity equations.

18 It can be seen that for small black holes the formulas in [8] allow $j_{2}$ to be non-zero with a bound similar to the one in 43]. 
coupling and strong coupling partition functions agree with each other (see 44 for an explanation).

It is possible that something similar will turn out to be true for the $\frac{1}{16}^{\text {th }}$ cohomology (see [1] for a possible mechanism). This makes the enumeration of the weakly coupled $Q$ cohomology an important problem. We hope to return to this problem in the near future.

\subsection{Enumeration of $\frac{1}{8}^{\text {th }}$, quarter and half BPS Cohomology}

In this subsection we will enumerate operators in the anti-chiral ring, i.e. operators that are annihilated by $Q^{\alpha 1}$, with $\alpha= \pm \frac{1}{2}$, and their Hermitian conjugates (these are the charges we called $Q$ and $Q^{\prime}$ in previous sections 19). All such states have $\Delta=0$ and $j_{1}=0$. It is not possible to isolate the contribution of these states to $\mathcal{I}_{Y M}$ (note the index lacks a chemical potential that couples to $j_{1}$ ); nonetheless we will be able to use dynamical information to count these states below.

This enumeration is easily performed in the free theory. Only the letters $X, Y, Z, \psi_{0, \pm,++}$ (see Table 2) and no derivatives contribute in this limit. We will denote these letters by $\bar{\Phi}_{i}(i=1 \ldots 3)$ and $\bar{W}_{\dot{\alpha}}(\dot{\alpha}= \pm)$ below. Note that these letters all have $j_{1}=0$ and $E=q_{1}+q_{2}+q_{3}$. The partition function

$$
Z_{c r-f r e e}=\operatorname{Tr} \exp \left[\sum_{i} \mu_{i} q_{i}+2 \zeta j_{2}\right]
$$

is given by the expression on the RHS of (5.2) with

$$
f_{B}=\sum_{i=1}^{3} e^{\mu_{i}}, \quad f_{F}=2 \cosh \zeta e^{\frac{\mu_{1}+\mu_{2}+\mu_{3}}{2}}
$$

Note that $1-f_{B}-f_{F}$ is positive at small $\mu_{i}$ but negative at large $\mu_{i}$. We conclude that the partition function (6.1]) undergoes the phase transition described in [2, 3] at finite values of the chemical potentials, and that its logarithm evaluates to an expression of order $N^{2}$ at small $\mu_{i}$.

We now turn to the weakly interacting theory. As explained in [5, 44], at any nonzero coupling no matter how small, the set of supersymmetric operators is given by all gauge invariant anti-chiral fields $\bar{\Phi}_{i}, \bar{W}_{\dot{\alpha}}$ modulo relations $\left[\bar{\Phi}_{i}, \bar{\Phi}_{j}\right]=\left[\bar{\Phi}_{i}, \bar{W}_{\dot{\alpha}}\right]=0$ and $\left\{\bar{W}_{\dot{\alpha}}, \bar{W}_{\dot{\beta}}\right\}=0$ (the first of these follows from $\partial_{\bar{\Phi}_{i}} \bar{W}=0$ where $\bar{W}$ is the superpotential).

19 If we had chosen states annihilated by $\bar{Q}_{1}^{\dot{\alpha}}$ we would have obtained the chiral ring. 
In general there can be corrections to these relations (see [44]). We assume that such corrections do not change the number of elements in the ring. In fact, if we go to the Coulomb branch of $\mathcal{N}=4$ we get a $U(1)^{N}$ theory with no corrections at the level of the two derivative action. The chiral primary operators at a generic point of this moduli space are the same as all the operators that we are going to count.

It is now easy to enumerate the states in the chiral ring. The relations in the previous paragraph ensure that all the basic letters commute or anticommute, and so may be simultaneously diagonalized, so we must enumerate all distinct polynomials of traces of diagonal letters. This is the same thing as enumerating all polynomials of the $3 N$ bosonic and $2 N$ fermionic eigenvalues that are invariant under the permutation group $S_{N}$. We may now formally substitute the eigenvalues $\bar{\phi}_{i}^{f}$ and $\bar{W}_{\dot{\alpha}}^{f}(f=1 \ldots N)$ with bosonic and fermionic creation operators $a_{i}^{f}$ and $w_{\dot{\alpha}}^{f}$; upon acting on the vacuum these produce states in the Hilbert space of $N$ particles, each of which propagates in the potential of a 3 dimensional bosonic and a 2 dimensional fermionic oscillator. The permutation symmetry ensures that the particles are identical bosons or fermions depending on how many fermionic oscillators are excited. As a consequence we conclude that the cohomological partition function is given by the coefficient of $p^{N}$ in

$$
\begin{aligned}
Z_{1 / 8}\left(p, \gamma_{1}, \gamma_{2}, \gamma_{3}, \zeta\right) & =\sum_{N=0}^{\infty} p^{N} Z_{N}\left(\gamma_{1}, \gamma_{2}, \gamma_{3}, \zeta\right)= \\
& =\prod_{n, m, r=0}^{\infty} \frac{\prod_{s= \pm 1}\left(1+p e^{s \zeta} e^{-(2 n+1) \gamma_{1}-(2 m+1) \gamma_{2}-(2 r+1) \gamma_{3}}\right)}{\left(1-p e^{-n 2 \gamma_{1}-m 2 \gamma_{2}-r 2 \gamma_{3}}\right)\left(1-p e^{\left.-(2 n+2) \gamma_{1}-(2 m+2) \gamma_{2}-(2 m+2) \gamma_{3}\right)}\right.}
\end{aligned}
$$

Further discussion on these 1/8 BPS states can be found in [10].

We may now, specialize both the free and the interacting cohomologies listed above to $\frac{1}{4}^{\text {th }}$ BPS cohomology by taking the limit $\gamma_{3} \rightarrow \infty$. The only letters that contribute in this limit are $\bar{\Phi}_{1}$ and $\bar{\Phi}_{2}(X, Y$ of Table 2$)$. The final result for the interacting cohomology may be written as

$$
Z_{1 / 4}\left(p, \gamma_{1}, \gamma_{2}\right)=\sum_{N=0}^{\infty} p^{N} Z_{N}\left(\gamma_{1}, \gamma_{2}\right)=\frac{1}{\prod_{n, m=0}^{\infty}\left(1-p e^{-n 2 \gamma_{1}-m 2 \gamma_{2}}\right)}
$$

For a more explicit construction of 1/4 BPS operators see 445] and references therein.

It is instructive to compare the $\gamma_{3} \rightarrow \infty$ limit (6.4) of (6.3) to the same limit of the partition function over $Q$ cohomology of the previous section that also simplifies dramatically in this limit. The only letters that contribute in this limit are $X, Y, \Psi_{+,++-}$(where the 
indices refer to $j_{1}, q_{1}, q_{2}, q_{3}$ charges). Further, it is easy to verify that $Q \Psi_{+,++-} \propto[X, Y]$. As a consequence the matrices $X$ and $Y$ should commute and may be diagonalized; furthermore the matrix $\psi$ must also be diagonal (so that $Q$ anihillates it). The cohomology in this limit is thus given by the partition function of $N$ particles in a 2 bosonic and one fermionic dimensional harmonic oscillator.

$$
Z=\sum_{N} p^{N} \operatorname{Tr}\left[y^{2 J_{1}} e^{-\gamma_{i} L_{i}}\right]=\prod_{n, m \geq 0} \frac{\left(1+p y e^{-2(n+1) \gamma_{1}-2(m+1) \gamma_{2}}\right)}{\left(1-p e^{-2 n \gamma_{1}-2 m \gamma_{2}}\right)}
$$

The Index $\mathcal{I}^{W L}$ over this cohomology is then computed by setting $y=-1$. At this special value, terms in the numerator with values $m, n$ cancel against terms in the denominator with $m+1, n+1$ leaving only

$\mathcal{I}_{Y M}^{W L}=\sum_{N} \mathcal{I}_{Y M}^{W L}(N)=\sum_{N} p^{N} \operatorname{Tr}_{N}\left[(-1)^{F} e^{-\gamma_{i} L_{i}}\right]=\frac{1}{(1-p) \prod_{n=1}^{\infty}\left(1-p e^{-n 2 \gamma_{1}}\right)\left(1-p e^{-n 2 \gamma_{2}}\right)}$

This is an exact formula for the $\gamma_{3} \rightarrow \infty$ limit of the index $\mathcal{I}_{Y M}^{W L}$. Multiplying it with $(1-p)$ and setting $p$ to unity, we recover the large $N$ result (4.7) (at $\left.\gamma_{3}=\infty\right)$.

It is also possible to further specialize (6.4) to the half BPS cohomology (of states annihilated by supercharges with $q_{1}=\frac{1}{2}$ ) by taking the further limit $\gamma_{2} \rightarrow \infty$ to obtain

$$
Z_{1 / 2}\left(p, \gamma_{1}\right)=\sum_{N=0}^{\infty} p^{N} Z_{N}\left(\gamma_{1}\right)=\frac{1}{\prod_{n=0}^{\infty}\left(1-p e^{-n 2 \gamma_{1}}\right)}
$$

Note that the free half BPS cohomology, interacting half BPS cohomology and the $\gamma_{2}, \gamma_{3} \rightarrow \infty$ limit of $\mathcal{I}_{Y M}^{W L}$ all coincide. On the other hand the free quarter BPS cohomology sees many more states than the interacting quarter BPS cohomology which, in turn, sees a larger effective number of states than the $\gamma_{3} \rightarrow \infty$ limit of the index. The last quantity, the index, receives contributions from $\bar{\Phi}_{1}, \bar{\Phi}_{2}$ and $\psi_{+, 0 ;++-}$, which are all the states in table two which have $L_{3}=0$. This index sees a smaller number of states as a consequence of cancellations involving the presence of the fermion $\psi_{+, 0 ;++-}$. Again, the $\frac{1}{8}^{\text {th }}$ BPS free cohomology sees more states than the interacting cohomology, which in turn sees more states than the index, with no restrictions on chemical potentials. More explicitly, we can see that for very large charges, or very small chemical potentials the entropy of (6.7) is that of $N$ harmonic oscillators, which correspond basically to the eigenvalues. Similarly, (6.4), and (6.3) give the entropy of $2 N$ and $3 N$ harmonic oscillators respectively. All these entropies are basically linear in $N$ in the large $N$ limit. The intuitive reason is that the matrices commute, and so do not taking advantage of the full non-abelian structure of the theory. 


\subsection{Protected double trace operator in the $\mathbf{2 0}$}

As an example of a practical application of the exact partition function over the chiral ring (derived in the previous subsection) and the index $\mathcal{I}_{Y M}$ (defined and computed in sections 3 and 4), in this subsection we will demonstrate that the scaling dimension of a particular double trace operator Yang Mills operator is protected.20

Consider $S U(N) \mathcal{N}=4$ Yang Mills theory. Let us first study the set of operators with quantum numbers $\left(q_{1}, q_{2}, q_{3}\right)=(3,1,1)$ and $j_{1}=j_{2}=0$. Using $\Delta \geq 0$ we conclude that such operators have $E \geq 5$; we will be interested in operators that saturate this equality. Let us first consider free Yang Mills theory. The set of all such operators is easy to list; we find

$$
\begin{aligned}
& \operatorname{Tr}\left[W_{\alpha} W^{\alpha}\right] \operatorname{Tr}\left[X^{2}\right], \quad \operatorname{Tr}\left[W_{\alpha} X\right] \operatorname{Tr}\left[W^{\alpha} X\right] \\
& \operatorname{Tr}\left[X^{2}\right] \operatorname{Tr}[X Y Z], \quad \operatorname{Tr}\left[X^{2}\right] \operatorname{Tr}[X Z Y], \quad \operatorname{Tr}[X Y] \operatorname{Tr}\left[X^{2} Z\right], \quad \operatorname{Tr}[X Z] \operatorname{Tr}\left[X^{2} Y\right] \\
& \operatorname{Tr}[Y Z] \operatorname{Tr}\left[X^{3}\right]
\end{aligned}
$$

Turning now to the interacting theory, we note that all but one of these operators belongs to the $1 / 8$ BPS chiral ring, and so has protected scaling dimension 21. Indeed it is not difficult to check that the appropriate coefficient in (6.3) (after subtracting the $U(1)$ part and the single trace contribution) is 6 implying that 6 of the 7 operators in (6.8) have protected dimensions. The unprotected operator in (6.8) is simply $O^{\prime}=\operatorname{tr}\left[X^{2}\right] \operatorname{tr}[X[Y, Z]]$.

Note that the operators studied in the previous paragraph have $\Delta=0, L_{1}=6, L_{2}=2$, $L_{3}=2, J_{2}=0$. Notice that states with quantum numbers $\left(q_{1}, q_{2}, q_{3}\right)=(5 / 2,1 / 2,1 / 2)$, $j_{1}=\frac{1}{2}, j_{2}=0$ share these values for $\Delta, L_{i}, j_{2}$; (and, moreover, are unique in this regard in the double trace sector of the $S U(N)$ theory). As a consequence we will now list all double trace operators in the free theory with these quantum numbers. They are

$$
\begin{array}{lr}
\operatorname{tr}\left[\psi_{+,-++} X\right] \operatorname{tr}\left[X^{2}\right], & \operatorname{tr}\left[\psi_{+,+-+} Y\right] \operatorname{tr}\left[X^{2}\right], \quad \operatorname{tr}\left[\psi_{+,++-} Z\right] \operatorname{tr}\left[X^{2}\right] \\
\operatorname{tr}\left[\psi_{+,+-+} X\right] \operatorname{tr}[X Y], & \operatorname{tr}\left[\psi_{+,++-} X\right] \operatorname{tr}[X Z]
\end{array}
$$

It follows from the discussion above that the contribution of double trace operators with $L_{1}=6, L_{2}=L_{3}=4, J_{2}=0$ to the index $\mathcal{I}_{Y M}$ is $(7-5) e^{-6 \gamma_{1}-2 \gamma_{2}-2 \gamma_{3}}=$ $2 e^{-6 \gamma_{1}-2 \gamma_{2}-2 \gamma_{3}}$. As $\mathcal{I}_{\mathcal{Y} \mathcal{M}}$ is not renormalized, it must be that 4 out of the 5 operators

20 This subsection was added in the revised version to answer a question raised by M. Bianchi.

21 In general the interacting operator with good scaling dimension will have a complicated form, admitting admixtures with single trace operators. 
listed in (6.9) are exactly protected. The single non protected operator is easily identified; at infinite $N$ it is the operator

$O=\left(\operatorname{tr}\left[\psi_{+,-++} X\right]+\operatorname{tr}\left[\psi_{+,+-+} Y\right]+\operatorname{tr}\left[\psi_{+,++-} Z\right]\right) \operatorname{tr}\left[X^{2}\right]=Q_{+\frac{1}{2}, 1} \operatorname{tr}[X \bar{X}+Y \bar{Y}+Z \bar{Z}] \operatorname{tr} X^{2}$

Note that $Q_{-\frac{1}{2}, 1} O \propto O^{\prime}$; we see that the the two non protected operators $O$ and $O^{\prime}$ are married together in the same long multiplet.

We have concluded that four double trace operators of the form (6.9) are exactly protected. At the end of this subsection we will demonstrate that while three of these four operators are $S U(1,2 \mid 3)$ descendants, a fourth is and $S U(1,2 \mid 3)$ primary. As we have explained in section 3 , the decomposition of the index $\mathcal{I}_{Y M}$ into characters of $S U(1,2 \mid 3)$ yields information about linear combinations of the number of short representations of the Yang Mills theory. In the case at hand, the existence of precisely one protected primary with these quantum numbers implies the existence of exactly one double trace $c c$ type representation with quantum numbers $q_{1}=2, q_{2}=q_{3}=0$, (or $R_{1}=R_{3}=0, R_{2}=2$ ), $j_{1}=j_{2}=0$ (such a representation has $E=4$ ). This is an operator of the schematic form $O_{I J} Q_{J K}-\frac{1}{6} \delta_{I K} O_{L J} Q_{J L}$ where $O_{I J}$ is the single trace operator in the $\mathbf{2 0}$ of $S O(6)$. (This form is schematic because this operator will mix with single trace operators, see for example [46]). Indeed this operator was studied in 447,48,49 and a proof that it is protected was was given in [50,51,52], based on the non-renormalization theorem in [53]. The arguments of this subsection may be regarded as an alternate proof of this non renormalization.

To complete this subsection we will now demonstrate that 3 of the operators in (6.9) are $S U(1,2 \mid 3)$ descendants. In fact the operators in question will turn out to be descendants of $1 / 2$ and $1 / 4$ BPS states in $b b$ representations. In other words, some of them result from the action of $S U(1,2 \mid 3)$ generators on conformal primaries which have lower conformal weight. So let us understand the protected $b b$ representations with $E=4$. One of them arises from the $1 / 2$ BPS chiral primary operator $\operatorname{tr}\left[X^{2}\right]^{2}$. We can now consider the $S U(1,2 \mid 3)$ descendants of it. By analyzing in more detail the action of the supercharges we find that two of the states in $(6.9)$ are $S U(1,2 \mid 3)$ descendants of $\operatorname{tr}\left[X^{2}\right]^{2}$. Another operator that we should consider is the 1/4 BPS double trace operator that is in the 84 (the protected nature of this operator follows from the partition function of chiral primary operators (6.3), or (6.4)-in other words, it gives rise to operators in the chiral ring). This operator has $S U(4)$ Dynkin labels $R=(2,0,2)$. It turns out that there is one $S U(1,2 \mid 3)$ descendant of the $\mathbf{8 4}$ with the quantum numbers appearing in (6.9), completing our demonstration. 


\subsection{Large $N$ limits and Phase Transitions}

In this subsection we will study the large $N$ limit of the partition functions (6.3), (6.4), (6.7). We will first briefly consider the limit $N \rightarrow \infty$ at fixed values of the chemical potentials, and show that in this limit these partition functions reproduce the supergravity answers (5.15). We will then turn to large $N$ limits in which the chemical potentials scale with $N$. We find that the formulas for $1 / 4$ and $1 / 8$ BPS states lead to large $N$ phase transitions. This phase transition is the Bose-Einstein condensation of the lowest mode, the ground state of the harmonic oscillators we had in the previous subsection.

In the $N \rightarrow \infty$ and fixed chemical potential the partition functions (6.7), (6.4), (6.3), become independent of $N$. This limit is most easily evaluated by multiplying the partition functions by $(1-p) 22$ and setting $p=1$. The entropy then grows as a gas of massless particles in one, two and three dimensions respectively.

For half BPS states we have [54]

$$
Z_{1 / 2}\left(\gamma_{1}\right)=\frac{1}{\prod_{n=0}^{\infty}\left(1-e^{-n 2 \gamma_{1}}\right)}
$$

Clearly, in the large $N$ limit, (6.11) may be thought of as the multiparticle partition function for a system of bosons with

$$
Z_{1 / 2-s p}=\sum_{n=1}^{\infty} e^{-2 n \gamma_{1}}=\frac{1}{1-e^{-2 \gamma_{1}}}-1
$$

note that (6.12) is the same as the supergravity result (5.15) in the limits $\gamma_{2} \rightarrow \infty, \gamma_{1} \rightarrow \infty$. Similarly the large $N$ limit of (6.4) is the multiparticle partition function for a system of bosons whose single particle partition function is

$$
Z_{1 / 4-s p}=\sum_{n, m} e^{-2 n \gamma_{1}-2 m \gamma_{2}}=\frac{1}{\left(1-e^{-2 \gamma_{1}}\right)\left(1-e^{-2 \gamma_{2}}\right)}-1
$$

which is the same as (5.15) in the limit $\gamma_{3} \rightarrow \infty$. In a similar fashion, in the large $N$ limit of (6.3) is precisely the multiparticle partition function (5.16) a system of bosons and fermions whose single particle partition functions are given by (5.15).

22 This step cancels the divergent contribution of the ground state of the harmonic oscillator in this limit. We will have a lot more to say about this below. 
We now turn to large $N$ limits of these partition functions in which we will allow the chemical potentials to scale with $N$. Let us start with the $1 / 2$ BPS case, and set $\gamma_{1}=\gamma$. This case does not have a phase transition. We write

$$
\log Z(\gamma, p)=-\sum_{n} \log \left(1-p e^{-2 n \gamma}\right) \sim-\frac{1}{2 \gamma} \int_{0}^{\infty} d x \log \left(1-p e^{-x}\right)
$$

We can first solve for $p$ by writing

$$
N=p \partial_{p} \log Z=\frac{1}{2 \gamma} \int_{0}^{\infty} d x \frac{p e^{-x}}{1-p e^{-x}}=-\frac{1}{2 \gamma} \log (1-p)
$$

We can now write $\widetilde{\beta} \equiv N 2 \gamma$. Then (6.15) is independent of $N$ and it has a solution for all values of $\widetilde{\beta}$. We can then write the partition function as

$$
\log Z_{N}(\gamma)=N\left\{\frac{1}{\widetilde{\beta}} \int_{0}^{\infty} d x \log \left[1-\left(1-e^{-\widetilde{\beta}}\right) e^{-x}\right]-\log \left(1-e^{-\widetilde{\beta}}\right)\right\}
$$

We see that this formula is of order $N$. There is no transition as a function of $\widetilde{\beta}$. For large values of $\widetilde{\beta} \ll 1$, it turns out that $(\underline{6.16})$ is independent of $N$ when expressed in terms of $\gamma$. This can be most easily seen by setting $p=1$ in (6.14). As expected the change in behavior happens at a temperature $(2 \gamma)^{-1} \sim N$ which is when the trace relations start being important. For very small $\widetilde{\beta}$ we find that (6.16) becomes $\log Z_{N} \sim N[-\log \widetilde{\beta}+1]$, which captures the large temperature behavior of $N$ harmonic oscillators plus an $1 / N$ ! statistical factor.

Let us now consider $1 / 4$ BPS states. Let us set $\gamma_{1}=\gamma_{2}=\gamma$. For sufficiently large temperatures we approximate the partition function as

$$
\log Z(\beta, p)=\sum_{n_{1}, n_{2}}-\log \left(1-p e^{-\left(n_{1}+n_{2}\right) 2 \gamma}\right) \sim \frac{1}{(2 \gamma)^{2}} \int_{0}^{\infty} d x x\left[-\log \left(1-p e^{-x}\right)\right]
$$

Now we find a new feature when we compute

$$
N=\frac{1}{(2 \gamma)^{2}} \int d x x \frac{p e^{-x}}{1-p e^{-x}}=\frac{1}{(2 \gamma)^{2}} P l[2, p]
$$

where $P l[2, p]$ is the PolyLog function. Now we see that for the lowest value of the chemical potential, $p=1$, we get

$$
N_{\max }=\frac{1}{(2 \gamma)^{2}} \frac{\pi^{2}}{6}
$$


Defining $\widetilde{\beta} \equiv 2 \gamma \sqrt{N}$ we see that there is a critical temperature, $\widetilde{\beta}_{c}^{2}=\frac{\pi^{2}}{6}$, at which there is a phase transition obtained by setting $N_{\max }=N$ in (6.19). At temperatures smaller than this temperature we have a Bose-Einstein condensation of the ground state of the harmonic oscillator. In this regime the free energy $Z_{N}(\beta)$ is given by $(6.17)$ with $p=1$. For higher temperatures we are supposed to solve for $p$ using (6.18) and then insert it in (6.17) to compute the free energy. We get

$$
\log Z_{N}\left(\widetilde{\beta}^{\prime}\right)=N\left\{\frac{1}{\widetilde{\beta}^{2}} \int_{0}^{\infty} d x x\left[-\log \left(1-p(\widetilde{\beta}) e^{-x}\right)\right]-\log p(\widetilde{\beta})\right\}
$$

where $p(\widetilde{\beta})$ is the solution to (6.18). The for large temperatures we have $\log Z_{N} \sim$ $N\left[-\log \widetilde{\beta}^{2}+1\right]$ which captures the entropy of $N$ 2-dimensional harmonic oscillators plus the $1 / N$ ! statistical factor. It is possible to see that at $\widetilde{\beta}_{c}$ we have a second order phase transition.

One can find similar results for the $1 / 8$ BPS states. We set $\gamma_{i}=\gamma$. In this case the rescaled temperature is given by $\widetilde{\beta}^{\prime}=2 \gamma N^{1 / 3}$. The results are similar. For low temperatures the answer is independent of $N$ and for high temperatures we have a free energy which is linear in $N$ and is a function of the rescaled temperature $\widetilde{\beta}^{\prime}$. Again there is a second order phase transition corresponding to the Bose-Einstein condensation of ground state of the harmonic oscillator. If we think of these harmonic oscillators as arising from D3 branes wrapping the $S^{3}$, then we could think of this condensation as responsible for the fact that the $S^{3}$ is contractible, in the spirit of the transition in [55]. It would be nice to see if this can be made more precise.

\section{Discussion}

In this paper we have considered an index that counts protected multiplets for general four dimensional superconformal field theories. This quantity captures all the information about protected multiplets that can be obtained purely from group theory.

We have focused on the applications of this index to the $\mathcal{N}=4$ Yang Mills theory. It is possible that (and would be interesting if) our index turns out to be a useful tool in the study of $\mathcal{N}=1$ and $\mathcal{N}=2$ superconformal theories as well.

Indices of the form that we have constructed have obvious counterparts in superconformal theories in $d=3,5$, and 6 . It is possible that some of these indices (whose theory we have not worked out in detail) could have interesting applications. 
The later half of this paper was devoted to a study of the supersymmetric states of $\mathcal{N}=4$ Yang Mills on $S^{3}$. We computed this index for this theory and found that it precisely agrees with the free supergravity spectrum when we take the large $N$ limit. The index, however, does not reflect the large entropy of BPS black holes in $A d S_{5}$. This is not a contradiction because there is no clear argument from the supergravity point of view which says that the black holes should contribute to the index.

A satisfactory Yang Mills accounting of the entropy of the BPS black holes of [6, 7, 8] remains an important outstanding problem. We have not even aware of a field theoretic understanding of a rather gross feature of these black holes; the fact that supersymmetric solutions are known only when certain special relation between the charges is obeyed.

We think it should be possible to use weakly coupled Yang Mills theory to count the entropy of BPS black holes in $A d S_{5} \times S^{5}$. In such a counting one will have to put in some extra information about the dynamics of the theory (over and above the superconformal algebra), see section 6 . In this connection it is encouraging that the counting of BPS states in the free theory (without the $(-1)^{F}$ ) has some qualitative agreement with the black hole results.

\section{Acknowledgments}

We would like to thank O. Aharony, D. Berenstein, M. Bianchi, R. Gopakumar, L. Grant, S. Lahiri, J. Marsano, K. Papadodimas, H. Reall, R. Roiban, G. Rossi, N. Seiberg, A. Strominger and M. Van Raamsdonk for useful discussions. The work of JM was supported in part by DOE grant \#DE-FG02-90ER40542. The work of SM and SR was supported in part by an NSF Career Grant PHY-0239626, DOE grant DE-FG0191ER40654, and a Sloan Fellowship. 


\section{Appendix A. The $d=4$ Superconformal Algebra}

\section{A.1. The Commutation Relations}

$$
\begin{aligned}
& {\left[\left(J_{1}\right)_{\beta}^{\alpha},\left(J_{1}\right)_{\delta}^{\gamma}\right]=\delta_{\beta}^{\gamma}\left(J_{1}\right)_{\delta}^{\alpha}-\delta_{\delta}^{\alpha}\left(J_{1}\right)_{\beta}^{\gamma}} \\
& {\left[\left(J_{2}\right)_{\dot{\beta}}^{\dot{\alpha}},\left(J_{2}\right)_{\dot{\delta}}^{\dot{\gamma}}\right]=\delta_{\dot{\beta}}^{\dot{\gamma}}\left(J_{2}\right)_{\dot{\delta}}^{\dot{\alpha}}-\delta_{\dot{\delta}}^{\dot{\alpha}}\left(J_{2}\right)_{\dot{\beta}}^{\dot{\gamma}}} \\
& {\left[\left(J_{1}\right)_{\beta}^{\alpha}, P^{\gamma \dot{\delta}}\right]=\delta_{\beta}^{\gamma} P^{\alpha \dot{\delta}}-(1 / 2) \delta_{\beta}^{\alpha} P^{\gamma \dot{\delta}}} \\
& {\left[\left(J_{1}\right)_{\beta}^{\alpha}, K_{\gamma \dot{\delta}}\right]=\delta_{\gamma}^{\alpha} K_{\beta \dot{\delta}}-(1 / 2) \delta_{\beta}^{\alpha} K_{\gamma \dot{\delta}}} \\
& {\left[\left(J_{2}\right)_{\dot{\beta}}^{\dot{\alpha}}, P^{\dot{\delta} \gamma}\right]=\delta_{\dot{\beta}}^{\dot{\delta}} P^{\dot{\alpha} \gamma}-(1 / 2) \delta_{\dot{\beta}}^{\dot{\alpha}} P^{\dot{\delta} \gamma}} \\
& {\left[\left(J_{2}\right)_{\dot{\beta}}^{\dot{\alpha}}, K_{\dot{\delta} \gamma}\right]=\delta_{\dot{\delta}}^{\dot{\alpha}} K_{\dot{\beta} \gamma}-(1 / 2) \delta_{\dot{\beta}}^{\dot{\alpha}} K_{\dot{\delta} \gamma}} \\
& {\left[H, P^{\alpha \dot{\beta}}\right]=P^{\alpha \dot{\beta}}} \\
& {\left[H, K^{\alpha \dot{\beta}}\right]=-K^{\alpha \dot{\beta}}} \\
& {\left[K_{\alpha \dot{\beta}}, P^{\gamma \dot{\delta}}\right]=\delta_{\dot{\beta}}^{\dot{\delta}}\left(J_{1}\right)_{\alpha}^{\gamma}+\delta_{\alpha}^{\gamma}\left(J_{2}\right)_{\dot{\beta}}^{\dot{\delta}}+\delta_{\dot{\beta}}^{\dot{\delta}} \delta_{\alpha}^{\gamma} H} \\
& {\left[\left(J_{1}\right)_{\beta}^{\alpha}, Q^{\gamma n}\right]=\delta_{\beta}^{\gamma} Q^{\alpha n}-(1 / 2) \delta_{\beta}^{\alpha} Q^{\gamma n}} \\
& {\left[\left(J_{2}\right)_{\beta}^{\alpha}, \bar{Q}_{n}^{\gamma}\right]=\delta_{\beta}^{\gamma} \bar{Q}_{n}^{\alpha}-(1 / 2) \delta_{\beta}^{\alpha} \bar{Q}_{n}^{\gamma}} \\
& {\left[K_{\alpha \dot{\beta}}, Q^{\gamma n}\right]=\delta_{\alpha}^{\gamma} \bar{S}_{\dot{\beta}}^{n}} \\
& {\left[P_{\alpha \dot{\beta}}, \bar{Q}_{n}^{\gamma}\right]=\delta_{\alpha}^{\gamma} S_{n \dot{\beta}}} \\
& {\left[H, Q^{\gamma n}\right]=\frac{1}{2} Q^{\gamma n}, \quad\left[H, \bar{Q}_{n}^{\dot{\alpha}}\right]=\frac{1}{2} \bar{Q}_{n}^{\dot{\alpha}}, \quad\left[H, S_{\alpha n}\right]=-\frac{1}{2} S_{\alpha n}, \quad\left[H, \bar{S}_{\dot{\alpha}}^{n}\right]=-\frac{1}{2} \bar{S}_{\dot{\alpha}}^{n}} \\
& {\left[r, Q^{\gamma n}\right]=Q^{\gamma n}, \quad\left[r, \bar{Q}_{n}^{\dot{\alpha}}\right]=-\bar{Q}_{n}^{\dot{\alpha}}, \quad\left[r, S_{\alpha i}\right]=-S_{\alpha i}, \quad\left[r, \bar{S}_{\dot{\alpha}}^{i}\right]=S_{\dot{\alpha}}^{i}} \\
& \left\{S_{\alpha i}, Q^{\beta j}\right\}=\delta_{i}^{j}\left(J_{1}\right)_{\alpha}^{\beta}+\delta_{\alpha}^{\beta} R_{i}^{j}+\delta_{i}^{j} \delta_{\alpha}^{\beta}\left(\frac{H}{2}+r \frac{4-m}{4 m}\right) \\
& \left\{Q^{\alpha m}, \bar{Q}_{m}^{\dot{\alpha}}\right\}=P^{\alpha \dot{\alpha}} \delta_{m}^{n} \\
& \left\{S_{\alpha m}, \bar{S}_{\dot{\alpha}}^{m}\right\}=K_{\alpha \dot{\alpha}} \delta_{m}^{n} \\
& {\left[R_{i}^{j}, Q^{\alpha p}\right]=\delta_{i}^{p} Q^{\alpha j}-(1 / m) \delta_{i}^{j} Q^{\alpha p}} \\
& {\left[R_{n}^{m}, R_{q}^{p}\right]=\delta_{q}^{m} R_{n}^{p}-\delta_{n}^{p} R_{q}^{m}}
\end{aligned}
$$

The Cartan generators are $\mathcal{H}, \mathcal{J}_{1}=\left(J_{1}\right)_{2}^{2}=-\left(J_{1}\right)_{1}^{1}, \mathcal{J}_{2}=\left(J_{2}\right)_{2}^{2}=-\left(J_{2}\right)_{1}^{1}, \mathcal{R}_{n}=R_{n}^{n}-$ $R_{n+1}^{n+1}$. While we have used script letters here, the Cartan generators above are the same as those used in the rest of the paper. The eigenvalue under $\mathcal{H}$ is the energy $E$, the eigenvalues under $\mathcal{J}_{1}, \mathcal{J}_{2}$ are the angular momenta $j_{1}, j_{2}$ and the eigenvalues under $\mathcal{R}_{i}$ are the R-charges $r_{i}$. Notice that in the way that we have defined the generators the 
commutation relations of the $J_{\mathrm{S}}$ and the $R \mathrm{~s}$ differ by a sign. For this reason, in the case of $m=2$, the structure of BPS states and null vectors is not symmetric under the exchange of the $J$ and the $R$ quantum numbers.

\section{A.2. An Oscillator Construction of the Algebra}

It is possible to find an explicit oscillator construction of this algebra following [56]. We introduce two sets of bosonic oscillators $a^{\alpha}, b^{\dot{\alpha}}, \alpha, \dot{\alpha}=1,2$ with adjoints $a_{\alpha}, b_{\dot{\alpha}}$. In addition, we introduce fermionic oscillators $\alpha^{n}$ with adjoints $\alpha_{n}, n=1 . .4$. As expected the $a$ and $b$ oscillators will transform as Lorentz spinors whereas the fermionic oscillators will transform in the fundamental representation of $S U(4)$. The generators of the superconformal group are now defined as below:

$$
\begin{aligned}
H & =\frac{1}{2}\left(a^{\alpha} a_{\alpha}+b^{\dot{\alpha}} b_{\dot{\alpha}}\right)+1 \\
\left(J_{1}\right)_{\beta}^{\alpha} & =a^{\alpha} a_{\beta}-\frac{1}{2} \delta_{\beta}^{\alpha} a^{\gamma} a_{\gamma} \\
\left(J_{2}\right)_{\dot{\beta}}^{\dot{\alpha}} & =b^{\dot{\alpha}} b_{\dot{\beta}}-\frac{1}{2} \delta_{\dot{\beta}}^{\dot{\alpha}} b^{\dot{\gamma}} b_{\dot{\gamma}} \\
P^{\alpha \dot{\alpha}} & =a^{\alpha} b^{\dot{\alpha}} \\
K_{\alpha \dot{\alpha}} & =a_{\alpha} b_{\dot{\alpha}} \\
R_{s}^{n} & =\alpha_{s} \alpha^{n}-\frac{1}{m} \delta_{s}^{n} \alpha_{t} \alpha^{t} \\
Q^{\alpha n} & =a^{\alpha} \alpha^{n} \\
\bar{Q}_{n}^{\dot{\alpha}} & =b^{\dot{\alpha}} \alpha_{n} \\
S_{\alpha n} & =a_{\alpha} \alpha_{n} \\
\bar{S}_{\dot{\alpha}}^{n} & =b_{\dot{\alpha}} \alpha^{n} \\
r & =\alpha_{n} \alpha^{n} \\
C & =b^{\dot{\alpha}} b_{\dot{\alpha}}-a^{\alpha} a_{\alpha}-\alpha_{n} \alpha^{n}
\end{aligned}
$$

While $C$ appears in the oscillator construction it commutes with all the generators of the algebra and is not really part of it. When we construct representations of the algebra using oscillators we fix the total value of $C$. 


\section{Appendix B. Algebraic Details Concerned with the Index}

\section{B.1. Superconformal Indices from Joining Rules}

As we have explained in section 2, any index is given by the sum (2.6) where the sum runs over representations of the algebra, and the coefficients $\alpha[i]$ are chosen such that $I$ evaluates to zero on every combination of short representations that can pair into a long representation.

The simplest indices for the superconformal algebra are given by $\alpha[i]=0$ for all $i \neq i_{0}$ for some specific $i_{o}$; this choice of $\alpha[i]$ defines an index only when the representation $i_{0}$ never makes an appearance on the right hand side of (2.16). An inspection of (2.16) and Table 2 shows that this is true of the representations of the form $\mathbf{b x}$ with $R_{1}=0$ or $R_{1}=1$ and representations of the form $\mathbf{x b}$ with any of $R_{m-1}=0$ or $R_{m-1}=1$. The number of all such representations constitutes an index.

We briefly pause to list these special representations in the most physically relevant cases $m=1,2,4$. Protected representations do not exist in the $\mathcal{N}=1$ algebra $(m=1)$. In the $\mathcal{N}=2$ algebra $(m=2)$ they consist of $S U(2)_{R}$ singlets with $j_{1}=0$ and $E=2 j_{2}+r / 2$, $S U(2)_{R}$ doublets with $j_{1}=0$ and $E=2 j_{2}+r / 2+2$ and chirality flips $\left(j_{1} \leftrightarrow j_{2}, r \leftrightarrow-r\right)$ of these. In the $\mathcal{N}=4(m=4)$ algebra they are representations with $R_{1}=0=j_{1}$ and $E=2 j_{2}+R_{2}+R_{3} / 2$, with $R_{1}=1, j_{1}=0$ and $E=2 j_{2}+3 / 2+R_{2}+R_{3} / 2$, and the chirality flips $\left(j_{1} \leftrightarrow j_{2}, R_{i} \leftrightarrow R_{4-i}\right)$ of these. Note that this includes representations with $j_{1}=j_{2}=R_{1}=R_{3}=0$ and $E=R_{2}$; these are the famous chiral primaries (gravitons) of the $\mathcal{N}=4$ theory.

Let us now turn to indices that have support on representations that do appear on the RHS of (2.16). We first consider indices built out of representations of the form $\widetilde{\mathbf{c a}}$. It follows from the first equation in (2.16) that, on an index

$$
\alpha\left[\widetilde{\mathbf{c}} \mathbf{a}_{j_{1}, j_{2}, r, R_{1}, R_{j}}\right]+\alpha\left[\widetilde{\mathbf{c}} \mathbf{a}_{j_{1}-\frac{1}{2}, j_{2}, r+1, R_{1}+1, R_{j}}\right]=0 .
$$

To begin with let us assume $m>1$. Notice that the two representations that appear in (B.1) have equal values of

$$
j_{2}, \quad r^{\prime} \equiv r-R_{1} \quad, M \equiv R_{1}+2 j_{1}, \quad R_{j} \quad(j=2 \ldots m-1) .
$$

The number of representations with given values for these conserved quantum number is $M+2$ (recall $j_{1}$ varies in half integer units from $-\frac{1}{2}$ upto $M / 2$; see Table 2 ). The $\alpha$ 
coefficients for these representations are constrained by $M+1$ equations. We conclude that there is exactly one index for any given set of charges (B.2) that obeys $E_{1}>E_{2}$; this index is given by (2.18).

In a very similar fashion, we conclude that (2.19) also defines an index provided $E_{2}>E_{1}$.

If $E_{1}=E_{2}$ the last equation in (2.16) applies. We have a total of $(M+2)(N+$ 2) representations with given values for $M, N, r^{\prime \prime \prime} \equiv r+2 j_{1}-2 j_{2}$ and $R_{l}$. The $\alpha^{\prime} s$ corresponding to these representations are constrained by $(M+1)(N+1)$ equations (from the last equation in (2.16)). This leaves us with an $M+2+N+2-1$ dimensional linear vector space of Indices. A convenient basis for these Indices is given by $N+2$ indices (2.18) (see the LHS of the equation below) ( plus the $M+2$ indices of the form (2.19), (see the RHS of the equation below) subject to the single additional constraint (2.20).

Finally we turn to the special case $m=1$ (the $\mathcal{N}=1$ algebra). As we have remarked above, no representations are absolutely protected in this case. The two indices (2.18) and (2.19), formally continue to be protected; however the expressions for these indices

$$
\begin{aligned}
I_{j_{2}, r^{\prime}} & =\sum_{p=-1}^{\infty}(-1)^{(p+1)} n\left[\widetilde{\mathbf{c}}_{\frac{p}{2}, j_{2}, r^{\prime}+p}\right] \\
I_{j_{1}, r^{\prime \prime}}^{R} & =\sum_{p=-1}^{\infty}(-1)^{p} n\left[\mathbf{x}_{j_{1}, \frac{p}{2}, r^{\prime \prime}-p}\right]
\end{aligned}
$$

(where $r^{\prime}=r+2 j_{1}$ and $r^{\prime \prime}=r-2 j_{2}$ ) now involves a sum over an infinite number of representations, and so could diverge.

\section{B.2. The Index $\mathcal{I}^{W L}$ as a sum over characters}

We define $Q^{\prime} \equiv Q^{\frac{1}{2}, 1}$, the $S U(2)$ partner of $Q \equiv Q^{-\frac{1}{2}, 1} \cdot Q^{\prime}$ has charge $\Delta=-2$. All other supercharges either have $\Delta=0(Q$ itself and all the supercharges in the $S U(1,2 \mid m-$ 1) subalgebra) or $\Delta=2$ (all other supercharges). There are also negative $\Delta$ bosonic generators. For example the $S U(2)$ spin operator $J_{1}^{+}$has $\Delta=-2$ and it appears in the anticommutation relation between $S$ and $Q^{\prime}$. We also get negative $\Delta$ states among the raising operators of $S U(m)$.

Notice that we can rederive some of the results in section two as follows. We start with the anticommutation relation (3.1). From this we derive that all states should obey $\Delta \geq 0$. Now suppose that we start with the highest weight state with Cartan charges 
$\left|\psi_{0}\right\rangle=\left|E, j_{1}, j_{2}, r, R_{i}\right\rangle$. This state has the lowest value of $\Delta$ among all the level zero states, which is $\Delta_{0}=E-\left(E_{1}-2\right)$, where $\left(E_{1}-2\right)$ is the combination of charges appearing in the right hand side of (3.1). Notice that we cannot lower the value of $\Delta$ by acting with bosonic generators since this is a highest weight state under the $S U(2) \times S U(2) \times S U(m)$ subalgebra. We now start acting with the supercharges $Q^{\alpha, i}$ on this state. When we act with $Q^{\prime}$ we lower the value of $\Delta$. If $Q^{\prime}$ does not annihilate the state we conclude that $\Delta_{0}-2 \geq 0$. If it is strictly bigger, then we have the representations of the generic type, which we called $\mathbf{a}$ in section two. When it becomes equal to zero, then we get representations of the type c, which obey $E=E_{1}$. The final possibility is that $Q^{\prime}$ annihilates the level zero state. Using the anticommutator of $Q^{\prime}$ and $S^{\prime}$ we notice that this can happen only if $\Delta_{0}+4 j_{1}=0$. Since, $\Delta_{0} \geq 0$, this implies that $j_{1}=0$ and $E=E_{1}-2$. So we have a representation called b in section two.

Using some of these ideas it is possible also to understand the structure of the null vectors. For that it is convenient to consider level one states of the form $J^{-} Q^{\prime}\left|\psi_{0}\right\rangle$ and $Q\left|\psi_{0}\right\rangle$. Using the anticommutation relations it is possible to show that the determinant of the $2 \times 2$ matrix of inner products among these states is proportional to $2 j_{1}\left(\Delta_{0}-2\right)$. So we find that in the case that $\Delta_{0}=2$ we have a null state. It is also easy to show that the state $Q^{\prime}\left|\psi_{0}\right\rangle$ has positive norm (if $\Delta_{0} \geq 2$ ). This latter state transforms in the representation with highest weights $\left(E+\frac{1}{2}, j_{1}+\frac{1}{2}, j_{2}, r+1, R_{1}+1, R_{j}\right)$. Thus the zero norm state we just mentioned transforms in the representation of the form $\left(E+\frac{1}{2}, j_{1}-\frac{1}{2}, j_{2}, r+1, R_{1}+1, R_{j}\right)$. This follows from the fact that we have one state with these quantum numbers plus the fact that if we had any other states with higher weights then we would be able to decrease the value of $\Delta$ for this state below 2 and we could change it in a continuous fashion (as we increase the energy of the original state away from the value that makes it a c type representation), but this is not possible. So we have recovered the statements in section two about the structure of the null vectors of the $\mathbf{c}$ type representations, at least for the case $j_{1}>0$. We can similarly continue the analysis for the structure of the null vectors of the c representations with $j_{1}=0$.

We can use some of these facts also to learn about the structure of the states that contribute to the index. For this purpose we should note that we get states with $\Delta=0$ by applying $Q^{\prime}$ to $\left|\psi_{0}\right\rangle$. This state has Cartan charges

$$
\left(E+\frac{1}{2}, j_{1}+\frac{1}{2}, j_{2}, r+1, R_{1}+1, R_{i}\right)
$$


It is also easy to see that it transforms in the $S U(1,2 \mid m-1)$ representation with charges $\left(E^{\prime}, j_{2}, r^{\prime}, R_{j}\right)$ given in terms of the map (3.4) applied to (B.4). In this way we obtain formula (3.5), for type $\mathbf{c}$ BPS representations. The factor $(-1)^{2 j_{1}+1}$ comes from the statistics factor associated to (B.4) which will not be included when we consider the character in the subalgebra, whose sign depends only on the $j_{2}$ quantum numbers.

For type $\mathbf{b}$ BPS representations the highest weight state itself has $\Delta=0$, so we find that $\left(E^{\prime}, j_{2}^{\prime}, r^{\prime}, R_{j}\right)$ are directly given by the formula in (3.4) in terms of the Cartan eigenvalues of $\left|\psi_{0}\right\rangle$ and we do not get any overall minus sign. This is summarized in (3.7).

The character on the $S U(1,2 \mid m-1)$ character in (3.5) manifestly depends on the quantum numbers $j_{1}, r, R_{1}$ of a $\widetilde{\mathbf{c}}$ representation only through the combination $j_{1}+r / 2$, $r-R_{1}$. This leads to $(3.8)$ and (3.9) and (3.7) for $\widetilde{\mathbf{c}}$ representations.

\section{B.3. Representation Theory of the Subalgebra $S U(2,1 \mid m-1)$}

The representation theory of the subalgebra is easily worked out, and closely mimics the pattern presented in the previous section. Briefly, representations are labeled by the quantum numbers $\left(E^{\prime}, j_{2}^{\prime}, r^{\prime}, R_{i}^{\prime}\right)$ that specify the $U(1) \times S U(2) \times U(m-1)(i=2 \ldots m-1)$ quantum numbers of the lowest weight state. Acting on this lowest weight state with the supersymmetries charged under $J_{2}^{\prime}$, we find a set of level one states; the lowest norm among these states occurs for those that transform in $\left(E^{\prime}+\frac{1}{2}, j_{2}^{\prime}-\frac{1}{2}, r^{\prime}-1, R_{j}^{\prime}, R_{m-2}^{\prime}+1\right)$ $(j=2 \ldots m-2)$; this norm is given by

\|\|$_{R, s u b}^{2}=E^{\prime}-3 j_{2}+3 \delta_{j_{2} 0}-3-3 \frac{\sum_{k=1}^{m-2} k R_{k}^{\prime}}{m-1}+\frac{r^{\prime}(4-m)}{(m-1)} \equiv E^{\prime}+3 \delta_{j_{2}, 0}-E_{2 s u b}^{\prime}\left(j_{2}^{\prime}, r^{\prime}, R_{i}^{\prime}\right)$.

Acting on the lowest weight states with supersymmetries uncharged under $j_{2}$, we find a set of states; the lowest norm occur for those states that transform in $\left(E^{\prime}+1, j_{2}, r^{\prime}+\right.$ $\left.1, R_{1}^{\prime}+1, R_{j}^{\prime}\right)(j=2 \ldots m-2)$. The norm of these states is given by

$$
\|\|_{s u b, L}^{2}=E^{\prime}-\frac{3}{2} \frac{\sum_{k=1}^{m-2}(m-1-k) R_{k}^{\prime}}{m-1}-\frac{r^{\prime}(4-m)}{2(m-1)} \equiv E-E_{1 s u b}^{\prime}\left(r^{\prime}, R_{i}^{\prime}\right) .
$$

Unitary representations occur when $E^{\prime} \geq E_{1 s u b}^{\prime}$ and $E^{\prime} \geq E_{2 s u b}^{\prime}$. When these inequalities are strictly satisfied, the representations are long and are denoted by $(\mathbf{a a})_{s u b}\left[E^{\prime}, j_{2}^{\prime}, r^{\prime}, R_{i}^{\prime}\right]$. Representations with $E^{\prime}=E_{2 s u b}^{\prime}$ are short, and are denoted by $(\mathbf{x c})_{s u b}\left[j_{2}^{\prime}, r^{\prime}, R_{i}^{\prime}\right]$. When $j_{2}^{\prime}=0$ the null states of this representation occur at level 2 . In addition, at $j_{2}^{\prime}=0$ we have 
a short representation at $E^{\prime}=E_{2 s u b}^{\prime}-3$, denoted by $(\mathbf{x b})_{s u b}\left[0, r^{\prime}, R_{i}^{\prime}\right]$. Representations with $E^{\prime}=E_{1 \text { sub }}^{\prime}$ are denoted by $(\mathbf{b x})_{\text {sub }}\left[j_{2}^{\prime}, r^{\prime}, R_{i}^{\prime}\right]$.

Now consider a representation $R$ of the full algebra that is of the form $\mathbf{c x}$. The highest weight state of $R$ has $\Delta=2$. Acting on this with $Q^{\prime}=Q^{+\frac{1}{2}, 1}$, we obtain a state with charges $\left(E+\frac{1}{2}, J_{1}+\frac{1}{2}, J_{2}, r+1, R_{1}+1, R_{i}\right)$. This state has $\Delta=0$ and serves as the highest weight of the representation $R^{\prime}$ of the subalgebra. If $R$ is of the form bx then, its highest weight state has $\Delta=0$ and also serves as the highest weight of $R^{\prime}$. If $R$ is of the form ax, then it has no states with $\Delta=0$.

Let us investigate if the representation $R^{\prime}$ so obtained satisfied the unitarity bounds from (B.5).

First, consider the case where $R$ is $\mathbf{c x}$. Then highest weight of $R^{\prime}$ is specified by the charges given by $\vec{c}$ in (3.7). Substituting these values of the charges into equation (B.5), (B.6) we find that

$$
\begin{aligned}
\|\|_{2}^{2} & =3 \delta_{0}^{j_{2}}+\frac{3}{2}\left(E_{1}-E_{2}\right) \\
\|\|_{1}^{2} & =3 j_{1}+3+\frac{3}{2} R_{1}
\end{aligned}
$$

So, $R^{\prime}$ is long unless $\left(E_{1}=E_{2}\right)$. In this case, $R \sim \mathbf{c c}$ and $R^{\prime}$ is short. If $j_{2}=0$ it is possible to have $E_{2}=E_{1}+2$, and then $R \sim \mathbf{c b}$ and $R^{\prime}$ is short.

Now, let $R=\mathbf{b x}$. Then

$$
\begin{aligned}
\|\|_{2}^{2} & =3 \delta_{0}^{j 2}-3+\frac{3}{2}\left(E_{1}-E_{2}\right) \\
\|\|_{1}^{2} & =\frac{3}{2} R_{1}
\end{aligned}
$$

If $\mathbf{x}$ is $\mathbf{a}$ or $\mathbf{c}$, we have $E_{1}-E_{2} \geq 2$. If this inequality is saturated, $R \sim \mathbf{b c}$ and $R^{\prime}$ is short. $R^{\prime}$ may also be short if $R_{1}=0$. Finally, when $j_{2}=0$ and $E_{1}=E_{2}, R \sim \mathbf{b b}$ and $R^{\prime}$ is short.

Using all of this, the decomposition of long representations as they hit unitarity bound follows immediately; we will not explicitly list the character formulae.

\section{Appendix C. Conventions and Computations for the $\mathcal{N}=4$ Index}

\section{C.1. Weights of the Supercharges}

In this subsection we list the weights of the supercharges under the Cartan elements $\left(E, J_{1}^{3}, J_{2}^{3}, R_{1}, R_{2}, R_{3}\right)$. 


$$
\begin{aligned}
Q_{ \pm}^{1} & \rightarrow\left\{\frac{1}{2}, \pm \frac{1}{2}, 0,1,0,0\right\} \\
Q_{ \pm}^{2} & \rightarrow\left\{\frac{1}{2}, \pm \frac{1}{2}, 0,-1,1,0\right\} \\
Q_{ \pm}^{3} & \rightarrow\left\{\frac{1}{2}, \pm \frac{1}{2}, 0,0,-1,1\right\} \\
Q_{ \pm}^{4} & \rightarrow\left\{\frac{1}{2}, \pm \frac{1}{2}, 0,0,0,-1\right\} \\
\bar{Q}_{1, \pm} & \rightarrow\left\{\frac{1}{2}, 0, \pm \frac{1}{2},-1,0,0\right\} \\
\bar{Q}_{2, \pm} & \rightarrow\left\{\frac{1}{2}, 0, \pm \frac{1}{2}, 1,-1,0\right\} \\
\bar{Q}_{3, \pm} & \rightarrow\left\{\frac{1}{2}, 0, \pm \frac{1}{2}, 0,1,-1\right\} \\
\bar{Q}_{4, \pm} & \rightarrow\left\{\frac{1}{2}, 0, \pm \frac{1}{2}, 0,0,1\right\}
\end{aligned}
$$

\section{C.2. Racah Speiser Algorithm}

The Racah Speiser algorithm is an efficient way to calculate tensor products. Consider a highest weight state $\mid \Lambda>$ and the complete set of states in another representation $\left|\lambda_{i}\right\rangle$. We denote the half sum of positive roots by $\rho$.

The Racah-Speiser algorithm tells us that to obtain the representations in the tensor product, we need to perform the following two steps.

1. First count all representations $\left|\Lambda+\lambda_{i}\right\rangle$, where $\Lambda+\lambda_{i}$ is in the fundamental Weyl Chamber[All weights are non-negative].

2. If $\Lambda+\lambda_{i}+\rho$ is on the boundary of the Weyl chamber i.e. at least one weight is zero, then throw away this representation. ( $\rho$ is the half-sum of the positive roots).

3. If $\Lambda+\lambda_{i}$ is not on the boundary of the Weyl Chamber, there exists a unique Weyl reflection, $\sigma$ such that $\sigma\left(\Lambda+\lambda_{i}+\rho\right)-\rho$ is in the Weyl Chamber. Count this representation with a plus or a minus sign depending on the sign of $\sigma$.

We use this algorithm to obtain the state contents tabulated below. It is interesting that for the Yang Mills multiplet, using the Racah Speiser algorithm automatically gives us the representations corresponding to the equations of motion with negative signs.

\section{C.3. State content of 'graviton' representations}

As explained in section 4.2 the spectrum of Type IIB supergravity compactified on $A d S_{5} \times S^{5}$ organizes into representations of the superconformal algebra that are are built 
on a lowest weight state that is a scalar in the $(n, 0,0)_{S O(6)}=(0, n, 0)_{S U(4)}$ representation of the R-symmetry group. When restricted to $\Delta=0$, they yield a short representation of the subalgebra that we shall call $S_{n}$. $S_{n}$ has lowest weight $\left[E=n, j_{2}=0, R_{2}=n, R_{3}=0\right]$. We may explicitly compute the $S U(2,1) \times S U(3)$ content of $S_{n}$ by starting with the lowest weight state, repeatedly acting on it with the $Q$ and $\bar{Q}$ operators, and deleting states of zero norm. This process is expedited by using the Racah Speiser algorithm explained in C.2.

In the table below we explicitly list the $S U(2,1) \times S U(3)$ content of $S_{n}$ using the notation $\left[E^{\prime}, j_{2}^{\prime}, R_{1}^{\prime}, R_{2}^{\prime}\right]$ where $\left[E^{\prime}, j_{2}^{\prime}\right]$ specify the weight of the lowest weight state under the compact $U(1) \times S U(2)$ subgroup of $S U(2,1)$ and $\left[R_{2}^{\prime}, R_{3}^{\prime}\right]$ are Dynkin labels for $S U(3)$. This can also be found by looking at the list of Kaluza Klein modes in [36].

\section{Table 3: Content of $S_{n}$}

\begin{tabular}{c|c|c|c}
$(-\mathbf{1})^{\mathbf{F}} \mathbf{E}^{\prime}$ & $\mathbf{J}_{\mathbf{2}}^{\prime}$ & $\mathbf{R}_{\mathbf{1}}^{\prime}$ & $\mathbf{R}_{\mathbf{2}}^{\prime}$ \\
\hline$n$ & 0 & $\mathrm{n}$ & 0 \\
$-\left(n+\frac{1}{2}\right)$ & $\frac{1}{2}$ & $\mathrm{n}-1$ & 0 \\
$n+1$ & 0 & $\mathrm{n}-2$ & 0 \\
$-(n+1)$ & 0 & $\mathrm{n}-1$ & 1 \\
$n+\frac{3}{2}$ & $\frac{1}{2}$ & $\mathrm{n}-2$ & 1 \\
$-(n+2)$ & 0 & $\mathrm{n}-3$ & 1 \\
$n+2$ & 0 & $\mathrm{n}-1$ & 0 \\
$-\left(n+\frac{5}{2}\right)$ & $\frac{1}{2}$ & $\mathrm{n}-2$ & 0 \\
$n+3$ & 0 & $\mathrm{n}-3$ & 0
\end{tabular}

For $n=2$ we just drop the lines containing $n-3$.

On the other hand, for $n=1$ we have further shortening and we find

\section{Table 4: Content of $S_{1}$}

\begin{tabular}{c|c|c|c}
$(-\mathbf{1})^{\mathbf{F}} \mathbf{E}^{\prime}$ & $\mathbf{J}_{\mathbf{2}}^{\prime}$ & $\mathbf{R}_{\mathbf{1}}^{\prime}$ & $\mathbf{R}_{\mathbf{2}}^{\prime}$ \\
\hline 1 & 0 & 1 & 0 \\
$-\frac{3}{2}$ & $\frac{1}{2}$ & 0 & 0 \\
-2 & 0 & 0 & 1 \\
3 & 0 & 0 & 0 \\
3 & 0 & 0 & 023
\end{tabular}

23 This term comes from the fermionic equation of motion, hence it counts with a positive sign 


\section{C.4. Character of $S U(3)$}

We wish to compute the quantity

$$
\chi_{\mathrm{R}}\left(\theta_{1}, \theta_{2}\right)=\operatorname{Tr}_{\mathrm{R}} \exp i\left(R_{1} \theta_{1}+R_{2} \theta_{2}\right),
$$

where $R_{1}$ and $R_{2}$ form the Cartan subalgebra of $S U(3)$. We denote the eigenvalues of the highest weight state of a representation, under the operators $R_{i}$, by $r_{i}$. Furthermore, we define $v_{1}=\exp -i \theta_{2}, v_{2}=\exp i \theta_{1}, v_{3}=\exp i\left(\theta_{2}-\theta_{1}\right)$ 2 2

Then, the character (C.2) is given by the Weyl Character Formula [57].

$$
\chi_{R_{1}, R_{2}}=\frac{\left|\begin{array}{ccc}
v_{1}^{R_{1}+1} & v_{2}^{R_{1}+1} & v_{3}^{R_{1}+1} \\
v_{1}^{-R_{2}-1} & v_{2}^{-} R_{2}-1 & v_{3}^{-} R_{2}-1 \\
1 & 1 & 1
\end{array}\right|}{\left|\begin{array}{ccc}
v_{1}^{1} & v_{2}^{1} & v_{3}^{1} \\
v_{1}^{-1} & v_{2}^{-1} & v_{3}^{-1} \\
1 & 1 & 1
\end{array}\right|}
$$

\section{C.5. Translation between bases}

$S O(6) \rightarrow S U(4)$

First, we show how to translate between $S O(6)$ and $S U(4)$ notation. Denote the $S O(6)$ Dynkin labels by $q_{1}, q_{2}, q_{3}$ and the $S U(4)$ Dynkin labels by $R_{1}, R_{2}, R_{3}$.

$$
\begin{aligned}
& q_{1}=\frac{R_{1}}{2}+R_{2}+\frac{R_{3}}{2} \\
& q_{2}=\frac{R_{1}}{2}+\frac{R_{3}}{2} \\
& q_{3}=\frac{R_{1}}{2}-\frac{R_{3}}{2}
\end{aligned}
$$

$H^{\prime}, R_{i}^{\prime} \rightarrow L_{i}$

Next, we show how to translate between the basis formed by the $L_{i}, J_{2}$ and the Cartan generators of the subalgebra $H+J_{1}, J_{2}, R_{1}^{\prime}, R_{2}^{\prime}$ defined above. Note that $R_{1}^{\prime}=R_{2}$, $R_{2}^{\prime}=R_{3}$. Moreover, recall that the $L_{i}$ are specified by (4.9) which we recapitulate here:

$$
L_{1}=E+q_{1}-q_{2}-q_{3}, L_{2}=E+q_{2}-q_{1}-q_{3}, L_{3}=E+q_{3}-q_{1}-q_{2}
$$

24 These are the weights of the fundamental representation of $S U(3)$ 
Under the condition $\Delta=0$ we find (denoting $H+J_{1}=H^{\prime}$ )

$$
\begin{aligned}
L_{1} & =\frac{2}{3} H^{\prime}+\frac{2}{3}\left(2 R_{1}^{\prime}+R_{2}^{\prime}\right) \\
L_{2} & =\frac{2}{3} H^{\prime}+\frac{2}{3}\left(-R_{1}^{\prime}+R_{2}^{\prime}\right) \\
L_{3} & =\frac{2}{3} H^{\prime}+\frac{2}{3}\left(-R_{1}^{\prime}-2 R_{2}^{\prime}\right)
\end{aligned}
$$

In turn this implies a relationship between the chemical potentials. If $\theta_{H^{\prime}} H^{\prime}+\theta_{1} R_{1}^{\prime}+$ $\theta_{2} R_{2}^{\prime}=\gamma_{1} L_{1}+\gamma_{2} L_{2}+\gamma_{3} L_{3}$ then,

$$
\begin{aligned}
\theta_{H^{\prime}} & =\frac{2}{3}\left(\gamma_{1}+\gamma_{2}+\gamma_{3}\right) \\
\theta_{1} & =\frac{2}{3}\left(2 \gamma_{1}-\gamma_{2}-\gamma_{3}\right) \\
\theta_{2} & =\frac{2}{3}\left(\gamma_{1}+\gamma_{2}-2 \gamma_{3}\right)
\end{aligned}
$$

C.6. Index on the Fock space

Let us say that we have the single particle index

$$
Z_{s p}=\sum_{i} x_{i}^{B}-\sum_{i} x_{i}^{F}
$$

where the index $i$ runs over all the bosons and all the fermions. Then the index for a multiparticle system is given by

$$
Z_{\text {Fock }}=\prod_{i} \frac{\left(1-x_{i}^{F}\right)}{\left(1-x_{i}^{B}\right)}=e^{\sum_{n} \frac{1}{n} Z_{s p}\left(x^{n}\right)}
$$

So we find (4.15).

\section{Appendix D. Comparison of the Cohomological Partition Function and the Index}

Let the number of states with charges $J_{1}, J_{2}, L_{i}$ be given by $e^{S\left(J_{1}, J_{2}, L_{i}\right)}$. Then

$$
\begin{aligned}
& Z_{\text {free }}=\sum_{J_{1}, J_{2}, L_{i}} \exp \left[S\left(J_{1}, J_{2}, L_{i}\right)-\sum_{i} \gamma_{i} L_{i}-2 \zeta J_{2}\right] \\
& \mathcal{I}_{Y M}^{W L}=\sum_{J_{1}, J_{2}, L_{i}} \exp \left[S\left(J_{1}, J_{2}, L_{i}\right)-\sum_{i} \gamma_{i} L_{i}-2 \zeta J_{2}\right](-1)^{2\left(J_{1}+J_{2}\right)}
\end{aligned}
$$


where we have set all chemical potentials that couple to charges outside $S U(2,1 \mid 3)$ to zero in $Z_{\text {free. }}$. Let

$$
\exp \left[N^{2} S_{\text {eff }}\left(\widetilde{j}_{1}, \gamma_{i}\right)\right]=\sum_{J_{2}, L_{i}} \exp \left[S\left(J_{1}, J_{2}, L_{i}\right)-\sum_{i} \gamma_{i} L_{i}-2 \zeta J_{2}\right]
$$

where $\widetilde{j}_{1} \equiv J_{1} / N^{2} \gg 1$ and $\gamma_{i} \ll 1$. Let us assume that $S_{\text {eff }}$ is independent of $N$ in the large $N$ limit. We certainly have this property in the free theory, and we expect it in the interacting $\mathcal{N}=4$ theory, but it does not have to hold for every theory. We can then rewrite (D.1) as

$$
\begin{aligned}
Z_{\text {free }} & =\sum_{J_{1}} \exp \left[N^{2} S_{\text {eff }}\left(\widetilde{j}_{1}, \zeta, \gamma_{i}\right)\right] \\
\mathcal{I}_{Y M}^{W L} & =\sum_{J_{1}} \exp \left[N^{2}\left\{S_{\text {eff }}\left(\widetilde{j}_{1}, \zeta+\pi i, \gamma_{i}\right)+2 i \pi \widetilde{j}_{1}\right\}\right]
\end{aligned}
$$

Let us assume that that at fixed values of $\zeta, \gamma_{i}$ has a maximum at $\tilde{j}=a\left(\theta, \gamma_{i}\right)$ and that

$$
\begin{aligned}
S_{\mathrm{eff}}\left(a+\delta, \zeta, \gamma_{i}\right) & \approx S_{0}-2 b^{2} \delta^{2} \\
S_{0} & =S_{\mathrm{eff}}\left(a, \zeta, \gamma_{i}\right) .
\end{aligned}
$$

The contribution of this saddle point to the partition function in the first line of (D.3) is easily estimated 25 by

$$
Z_{\text {free }} \approx \sqrt{\frac{2 \pi}{b^{2} N^{2}}} \exp \left[N^{2} S_{0}\right]
$$

An estimation of the Index in the second line of (D.3) is a more delicate task as the summand changes by large values over integer spacings. To proceed we will assume that $S_{\text {eff }}\left(j_{1}, \zeta, \gamma_{i}\right)$ is a continuous function; i.e. that it does not evaluate to discontinuously different answers for integral and half integral values of $J_{1}$. This is a nontrivial assumption, which we believe to be true for free Yang Mills theory, but will not always be true in every theory. Under this assumption we will now estimate the contribution of the saddle point

25 For instance one could convert the sum into an integral using the Euler McLaurin formula [58] and approximate the integral using saddle points. A more careful estimate may be obtained by Poisson resumming, see the next paragraph. 
at $\widetilde{j}_{1}=a$ to the index by

$$
\begin{aligned}
\mathcal{I}_{Y M}^{W L} & =e^{N^{2} S_{0}} \sum_{m=-\infty}^{\infty} \exp \left[-\frac{b^{2} m^{2}}{2 N^{2}}+\pi i m\right] \\
& =e^{N^{2} S_{0}} \sum_{k=-\infty}^{\infty} \sqrt{\frac{2 \pi}{b^{2} N^{2}}} \exp \left[\frac{N^{2}(2 \pi)^{2}}{2 b^{2}}\left(k-\frac{1}{2}\right)^{2}\right] \\
& \approx 2 \sqrt{\frac{2 \pi}{b^{2} N^{2}}} \exp \left[N^{2}\left(S_{0}-\frac{\pi^{2}}{2 b^{2}}\right)\right]
\end{aligned}
$$

where we have used the Poisson resummation formula to go from the first to the second line of $(\underline{D .6})$.

Note that the contribution of the saddle point at $\widetilde{j}_{1}=a$ to the index is supressed compared to its contribution to the partition function. Moreover, if $S_{0}<\pi^{2} / 2 b^{2}$, the contribution of this saddle point is formally of order $e^{-a N^{2}}$; which means that the neighborhood of the saddle point does not contribute significantly to the index in the large $N$ limit; the Index receives its dominant contributions from other regions of the summation domain. A estimation from formulas of (5.6), (5.9) puts us in this regime

As a toy example of the suppression described in the last two paragraphs, consider the two identities

$$
\begin{aligned}
& Z=(2+1)^{N}=\sum_{k} 2^{k} \frac{N !}{k !(N-k) !} \\
& I=(2-1)^{N}=\sum_{k} 2^{k} \frac{N !}{k !(N-k) !}(-1)^{N-k} .
\end{aligned}
$$

The summation over $k$ in the first of (D.7) may be approximated by the integral

$$
\int_{x=0}^{1} e^{N \ln \frac{2^{x}}{x^{x}(1-x)^{1-x}}},
$$

which localizes around the saddle point value $x^{s}=\frac{2}{3}$ at large $N$, yielding $Z=3^{N}$. The contribution to $I$ from this saddle point, on the other hand, is proportional to $e^{N\left(\ln 3-\frac{\pi^{2}}{3}\right)}$, and so is utterly negligible, consistent with the fact that $I$ evaluates to unity. 26

26 Actually, a computation very similar to this toy example explains why the index grows more slowly that exponentially with energy in the 'low temperature phase' (while the cohomological partition function displays exponential growth in the same phase). The number of states that contribute at energy $E$ to the index is given by the coefficient of $x^{E}$ in (4.7). This is given by a multinomial expansion. When we weight the sum with $(-1)^{F}$, the multinomial sum stops growing exponentially just like (D.7) above. Hence, the Index never goes through a Hagedorn like transition. 


\section{References}

[1] E. Witten, "Constraints On Supersymmetry Breaking," Nucl. Phys. B 202, 253 (1982).

[2] B. Sundborg, "The Hagedorn transition, deconfinement and $N=4$ SYM theory," Nucl. Phys. B 573, 349 (2000) arXiv:hep-th/9908001.

[3] O. Aharony, J. Marsano, S. Minwalla, K. Papadodimas and M. Van Raamsdonk, "The Hagedorn / deconfinement phase transition in weakly coupled large N gauge theories," Adv. Theor. Math. Phys. 8, 603 (2004) arXiv:hep-th/0310285].

[4] E. Witten, "Anti-de Sitter space and holography," Adv. Theor. Math. Phys. 2, 253 (1998) arXiv:hep-th/9802150.

[5] O. Aharony, unpublished.

[6] J. B. Gutowski and H. S. Reall, "Supersymmetric AdS(5) black holes," JHEP 0402, 006 (2004) arXiv:hep-th/0401042.

[7] J. B. Gutowski and H. S. Reall, "General supersymmetric AdS(5) black holes," JHEP 0404, 048 (2004) arXiv:hep-th/0401129.

[8] Z. W. Chong, M. Cvetic, H. Lu and C. N. Pope, "General non-extremal rotating black holes in minimal five-dimensional gauged supergravity," arXiv:hep-th/0506029.

[9] A. Strominger and C. Vafa, "Microscopic Origin of the Bekenstein-Hawking Entropy," Phys. Lett. B 379, 99 (1996) arXiv:hep-th/9601029].

[10] D. Berenstein, "Large N BPS states and emergent quantum gravity," arXiv:hepth/0507203.

[11] C. Romelsberger, "An index to count chiral primaries in $\mathrm{N}=1 \mathrm{~d}=4$ superconformal field theories," arXiv:hep-th/0510060.

[12] G. Mack, "All Unitary Ray Representations Of The Conformal Group SU(2,2) With Positive Energy," Commun. Math. Phys. 55, 1 (1977).

[13] A. Barabanschikov, L. Grant, L. L. Huang and S. Raju, "The spectrum of Yang Mills on a sphere," arXiv:hep-th/0501063.

[14] S. Minwalla, "Restrictions imposed by superconformal invariance on quantum field theories," Adv. Theor. Math. Phys. 2, 781 (1998) arXiv:hep-th/9712074.

[15] V. G. Kac, "Lie Superalgebras," Adv. Math. 26, 8 (1977).

[16] V. K. Dobrev and V. B. Petkova, "All Positive Energy Unitary Irreducible Representations Of Extended Conformal Supersymmetry," Phys. Lett. B 162, 127 (1985).

[17] V. K. Dobrev and V. B. Petkova, "On The Group Theoretical Approach To Extended Conformal Supersymmetry: Classification Of Multiplets," Lett. Math. Phys. 9, 287 (1985).

[18] V. K. Dobrev and V. B. Petkova, "All positive energy unitary irreducible representations of the extended conformal superalgebra," Talk at the Symposium on Conformal Groups and Structures (Clausthal, 1985); Proceedings, eds. A.O. Barut and 
H.D. Doebner, Lecture Notes in Physics, Vol. 261 (Springer-Verlag, Berlin, 1986) pp. 300-308.

[19] V. K. Dobrev and V. B. Petkova, "Group Theoretical Approach To Extended Conformal Supersymmetry: Function Space Realizations And Invariant Differential Operators," Fortsch. Phys. 35, 537 (1987).

[20] F. A. Dolan and H. Osborn, "On short and semi-short representations for four dimensional superconformal symmetry," Annals Phys. 307, 41 (2003) arXiv:hepth/0209056].

[21] V. K. Dobrev, "Characters of the positive energy UIRs of D $=4$ conformal supersymmetry," arXiv:hep-th/0406154.

[22] K. Dasgupta, M. M. Sheikh-Jabbari and M. Van Raamsdonk, "Protected multiplets of M-theory on a plane wave," JHEP 0209, 021 (2002) arXiv:hep-th/0207050.

[23] N. Kim and J. H. Park, "Superalgebra for M-theory on a pp-wave," Phys. Rev. D 66, 106007 (2002) arXiv:hep-th/0207061.

[24] H. Lin and J. Maldacena, "Fivebranes from gauge theory," arXiv:hep-th/0509235.

[25] L. Motl, A. Neitzke and M. M. Sheikh-Jabbari, "Heterotic plane wave matrix models and giant gluons," JHEP 0306, 058 (2003) arXiv:hep-th/0306051.

[26] H. Liu, "Fine structure of Hagedorn transitions," arXiv:hep-th/0408001.

[27] L. Alvarez-Gaume, C. Gomez, H. Liu and S. Wadia, "Finite temperature effective action, AdS(5) black holes, and 1/N expansion," Phys. Rev. D 71, 124023 (2005) arXiv:hep-th/0502227.

[28] O. Aharony, J. Marsano, S. Minwalla and T. Wiseman, "Black hole - black string phase transitions in thermal 1+1 dimensional supersymmetric Yang-Mills theory on a circle," Class. Quant. Grav. 21, 5169 (2004) arXiv:hep-th/0406210.

[29] O. Aharony, J. Marsano, S. Minwalla, K. Papadodimas and M. Van Raamsdonk, "A first order deconfinement transition in large N Yang-Mills theory on a small $\mathrm{S}^{* *} 3$," Phys. Rev. D 71, 125018 (2005) arXiv:hep-th/0502149.

[30] P. Basu and S. R. Wadia, "R-charged AdS(5) black holes and large N unitary matrix models," arXiv:hep-th/0506203.

[31] O. Aharony, J. Marsano, S. Minwalla, K. Papadodimas, M. Van Raamsdonk and T. Wiseman, "The phase structure of low dimensional large $\mathrm{N}$ gauge theories on tori," arXiv:hep-th/0508077.

[32] J. F. Morales and H. Samtleben, "Higher spin holography for SYM in d dimensions," Phys. Lett. B 607, 286 (2005) arXiv:hep-th/0411246].

[33] M. Bianchi, J. F. Morales and H. Samtleben, "On stringy AdS(5) x S**5 and higher spin holography," JHEP 0307, 062 (2003) arXiv:hep-th/0305052.

[34] N. Beisert, M. Bianchi, J. F. Morales and H. Samtleben, "On the spectrum of AdS/CFT beyond supergravity," JHEP 0402, 001 (2004) arXiv:hep-th/0310292. 
[35] N. Beisert, M. Bianchi, J. F. Morales and H. Samtleben, "Higher spin symmetry and N = 4 SYM," JHEP 0407, 058 (2004) [arXiv:hep-th/0405057.

[36] M. Gunaydin and N. Marcus, "The Spectrum Of The $\mathrm{S}^{* *} 5$ Compactification Of The Chiral $\mathrm{N}=2, \mathrm{D}=10$ Supergravity And The Unitary Supermultiplets Of U(2, 2/4)," Class. Quant. Grav. 2, L11 (1985).

[37] M. B. Green, J. H. Schwarz and E. Witten, "Superstring Theory. Vol. 2: Loop Amplitudes, Anomalies And Phenomenology," Chapter 12, Cambridge University Press (1987).

[38] S. S. Gubser, I. R. Klebanov and A. W. Peet, "Entropy and Temperature of Black 3-Branes," Phys. Rev. D 54, 3915 (1996) arXiv:hep-th/9602135.

[39] A. Mikhailov, "Giant gravitons from holomorphic surfaces," JHEP 0011, 027 (2000) arXiv:hep-th/0010206.

[40] J. McGreevy, L. Susskind and N. Toumbas, "Invasion of the giant gravitons from anti-de Sitter space," JHEP 0006, 008 (2000) arXiv:hep-th/0003075.

[41] D. Berenstein, C. P. Herzog and I. R. Klebanov, "Baryon spectra and AdS/CFT correspondence," JHEP 0206, 047 (2002) arXiv:hep-th/0202150.

[42] E. Barnes, E. Gorbatov, K. Intriligator and J. Wright, "Current correlators and AdS/CFT geometry," arXiv:hep-th/0507146.

[43] J. C. Breckenridge, R. C. Myers, A. W. Peet and C. Vafa, "D-branes and spinning black holes," Phys. Lett. B 391, 93 (1997) arXiv:hep-th/9602065.

[44] F. Cachazo, M. R. Douglas, N. Seiberg and E. Witten, "Chiral rings and anomalies in supersymmetric gauge theory," JHEP 0212, 071 (2002) arXiv:hep-th/0211170.

[45] E. D'Hoker, P. Heslop, P. Howe and A. V. Ryzhov, "Systematics of quarter BPS operators in N = 4 SYM," JHEP 0304, 038 (2003) [arXiv:hep-th/0301104.

[46] M. Bianchi, B. Eden, G. Rossi and Y. S. Stanev, "On operator mixing in N $=4$ SYM," Nucl. Phys. B 646, 69 (2002) arXiv:hep-th/0205321]. M. Bianchi, G. Rossi and Y. S. Stanev, "Surprises from the resolution of operator mixing in N = 4 SYM," Nucl. Phys. B 685, 65 (2004) arXiv:hep-th/0312228.

[47] G. Arutyunov, S. Frolov and A. Petkou, "Perturbative and instanton corrections to the OPE of CPOs in N = 4 SYM(4)," Nucl. Phys. B 602, 238 (2001) [Erratum-ibid. B 609, 540 (2001)] arXiv:hep-th/0010137.

[48] M. Bianchi, S. Kovacs, G. Rossi and Y. S. Stanev, "Properties of the Konishi multiplet in N = 4 SYM theory," JHEP 0105, 042 (2001) arXiv:hep-th/0104016.

[49] B. Eden, C. Jarczak, E. Sokatchev and Y. S. Stanev, "Operator mixing in N = 4 SYM: The Konishi anomaly revisited," Nucl. Phys. B 722, 119 (2005) arXiv:hepth/0501077.

[50] B. Eden, A. C. Petkou, C. Schubert and E. Sokatchev, "Partial non-renormalisation of the stress-tensor four-point function in N $=4$ SYM and AdS/CFT," Nucl. Phys. B 607, 191 (2001) arXiv:hep-th/0009106. 
[51] G. Arutyunov, B. Eden, A. C. Petkou and E. Sokatchev, "Exceptional non-renormalization properties and OPE analysis of chiral four-point functions in N $=4 \operatorname{SYM}(4)$," Nucl. Phys. B 620, 380 (2002) arXiv:hep-th/0103230.

[52] G. Arutyunov, S. Frolov and A. C. Petkou, "Operator product expansion of the lowest weight CPOs in N = 4 SYM(4) at strong coupling," Nucl. Phys. B 586, 547 (2000) [Erratum-ibid. B 609, 539 (2001)] arXiv:hep-th/0005182.

[53] B. Eden, A. C. Petkou, C. Schubert and E. Sokatchev, "Partial non-renormalisation of the stress-tensor four-point function in $\mathrm{N}=4$ SYM and AdS/CFT," Nucl. Phys. B 607, 191 (2001) arXiv:hep-th/0009106.

[54] S. Corley, A. Jevicki and S. Ramgoolam, "Exact correlators of giant gravitons from dual N = 4 SYM theory," Adv. Theor. Math. Phys. 5, 809 (2002) arXiv:hepth/0111222].

[55] B. R. Greene, D. R. Morrison and A. Strominger, "Black hole condensation and the unification of string vacua," Nucl. Phys. B 451, 109 (1995) arXiv:hep-th/9504145.

[56] I. Bars and M. Gunaydin, "Unitary Representations Of Noncompact Supergroups," Commun. Math. Phys. 91, 31 (1983).

[57] J. Fuchs and C. Schweigert, "Symmetries, Lie algebras and representations: A graduate course for physicists,", Cambridge University Press (1997).

[58] G. B. Arfken and H. J. Weber, "Mathematical methods for physicists", Academic Press(2001). 